\title{
LOCAL TIMES AND SAMPLE PATH PROPERTIES OF THE ROSENBLATT PROCESS
}

\author{
GEORGE KERCHEV, IVAN NOURDIN, EERO SAKSMAN, AND LAURI VIITASAARI
}

\begin{abstract}
Let $Z=\left(Z_{t}\right)_{t \geq 0}$ be the Rosenblatt process with Hurst index $H \in$ $(1 / 2,1)$. We prove joint continuity for the local time of $Z$, and establish Hölder conditions for the local time. These results are then used to study the irregularity of the sample paths of $Z$. Based on analogy with similar known results in the case of fractional Brownian motion, we believe our results are sharp. A main ingredient of our proof is a rather delicate spectral analysis of arbitrary linear combinations of integral operators, which arise from the representation of the Rosenblatt process as an element in the second chaos.
\end{abstract}

Mathematics Subject Classifications (2010): Primary 60G18; Secondary 60J55.

Keywords: Rosenblatt process, Local times, Fourier transform, Hilbert-Schmidt operator.

\section{Introduction}

The Rosenblatt process $Z=\left(Z_{t}\right)_{t \geq 0}$ is a self-similar stochastic process with longrange dependence and heavier tails than those of the normal distribution. It depends on a parameter $H \in\left(\frac{1}{2}, 1\right)$ which is fixed in what follows. The process $Z$ belongs to the family of Hermite processes that naturally arise as limits of normalized sums of long-range dependent random variables [6]. The first Hermite process is the fractional Brownian motion, which is Gaussian and thus satisfies many desirable properties. The Rosenblatt process is the second Hermite process: it is no longer Gaussian and was introduced by Rosenblatt in [19]. Although less popular than fractional Brownian motion, the Rosenblatt process is attracting increasing interest in the literature, mainly due to its self-similarity, stationarity of increments, and long-range dependence properties. See for example [1, 5, 13, 16] for recent studies on different aspects of this process.

The Rosenblatt process $\left(Z_{t}\right)_{t \geq 0}$ admits the following stochastic representation, also known as the spectral representation:

$$
Z_{t}=\int_{\mathbb{R}^{2}} H_{t}(x, y) Z_{G}(d x) Z_{G}(d y) .
$$

In (1.1), the double Wiener-Itô integral is taken over $x \neq \pm y$ and $Z_{G}(d x)$ is a complex-valued random white noise with control measure $G$ satisfying $G(t A)=$ $|t|^{1-H} G(A)$ for all $t \in \mathbb{R}$ and Borel sets $A \in \mathbb{R}$ and $G(d x)=|x|^{-H} d x$. The kernel 
$H_{t}(x, y)$ is given by

$$
H_{t}(x, y)=\frac{e^{i t(x+y)}-1}{i(x+y)},
$$

and is a complex valued Hilbert-Schmidt kernel satisfying $H_{t}(x, y)=H_{t}(y, x)=$ $\overline{H_{t}(-x,-y)}$ and $\int_{\mathbb{R}^{2}}\left|H_{t}(x, y)\right|^{2} G(d x) G(d y)<\infty$. In particular, the spectral theorem applies, see [6], and allows one to write

$$
Z_{t} \stackrel{d}{=} \sum_{k=1}^{\infty} \lambda_{k}\left(X_{k}^{2}-1\right)
$$

where $\left(X_{k}\right)_{k \geq 1}$ is a sequence of independent standard Gaussian random variables and $\left(\lambda_{k}\right)_{k>1}$ are the eigenvalues (repeated according to the possible multiplicity) of the self-adjoint operator $A: L_{G}^{2}(\mathbb{R}) \rightarrow L_{G}^{2}(\mathbb{R})$, given by

$$
(A f)(x):=\int_{\mathbb{R}} H_{t}(x,-y) h(y) G(d y)=\int_{\mathbb{R}} H_{t}(x,-y) h(y)|y|^{-H} d y .
$$

Furthermore, $\sum_{k \geq 1} \lambda_{k}^{2}<\infty$ and thus (1.3) converges.

The goal of the present paper is to study the occupation density (also known as the local time) $L(x, I)$ of $Z$, where $x \in \mathbb{R}$ and $I \subset[0, \infty)$ is a finite interval. Recall that for a deterministic function $f: \mathbb{R}_{+} \rightarrow \mathbb{R}$, the occupation measure of $f$ is defined by

$$
\nu(A, B)=\mu\left(B \cap f^{-1}(A)\right),
$$

where $A \subset \mathbb{R}$ and $B \subset \mathbb{R}_{+}$are Borel sets and $\mu$ is the Lebesgue measure on $\mathbb{R}_{+}$. Observe that $\nu(A, B)$ represents the amount of time during a period $B$ where $f$ takes value in $A$. Then, when $\nu(\cdot, B)$ is absolutely continuous with respect to $\mu$, the occupation density (or local time) is given by the Radon-Nikodym derivative

$$
L(x, B)=\frac{d \nu}{d \mu}(x, B) .
$$

We study the local time via the analytic approach initiated by Berman [3. The idea is to relate properties of $L(x, B)$ with the integrability properties of the Fourier transform of $f$. Recall the following key result [8]:

Proposition 1.1. The function $f$ has an occupation density $L(x, B)$ for $x \in \mathbb{R}, B \in$ $\mathcal{B}([u, U])$ which is square integrable in $x$ for every fixed $B$ iff

$$
\int_{\mathbb{R}}\left|\int_{u}^{U} \exp (i \xi f(t)) d t\right|^{2} d \xi<\infty .
$$

\footnotetext{
${ }^{1}$ We shall actually later work more with the singular value sequence, which for a compact selfadjoint operator coincides with sequence of the absolute values of the eigenvalues in a decreasing order, and each one repeated according to multiplicity. Singular values have the advantage that they can be defined also for non-self-adjoint operators and satisfy very handy inequalities.
} 
Moreover, in this case, the occupation density can be represented as

$$
L(x, B)=\frac{1}{2 \pi} \int_{\mathbb{R}} \int_{B} \exp (i \xi(x-f(s))) d \xi d s .
$$

The deterministic function $f(t)$ can be chosen to be the single path of a stochastic process $\left(X_{t}\right)_{t \geq 0}$. To show almost sure existence and square integrability of $L(x, B)$ in that case, it will be enough to show that

$$
\mathbb{E}\left[\int_{\mathbb{R}}\left|\int_{u}^{U} \exp \left(i \xi X_{t}\right) d t\right|^{2} d \xi\right]<\infty,
$$

or equivalently

$$
\int_{\mathbb{R}} \int_{u}^{U} \int_{u}^{U} \mathbb{E}\left[\exp \left(i \xi\left(X_{s}-X_{t}\right)\right)\right] d s d t d \xi<\infty .
$$

If $\left(X_{t}\right)_{t \geq 0}$ is Gaussian, then one can evaluate $\mathbb{E}\left[\exp \left(i \xi\left(X_{s}-X_{t}\right)\right)\right]$ explicitly to establish (1.4). It leads to the well-known Gaussian criterion:

Proposition 1.2 (Lemma 19 in [8]). Suppose that $X$ is Gaussian and centered, and satisfies

$$
\int_{[u, U]^{2}} \Delta(s, t)^{-1 / 2} d s d t<\infty,
$$

where $\Delta(s, t)=\mathbb{E}\left[\left(X_{s}-X_{t}\right)^{2}\right]$. Then $\left(X_{t}\right)_{t \geq 0}$ has an occupation density $L=$ $L(x, B, \omega)$ which, for $B$ fixed, is $P$-a.s. square integrable in $x$.

In our setting $\left(X_{t}\right)_{t \geq 0}=\left(Z_{t}\right)_{t \geq 0}$ is the Rosenblatt process which is not Gaussian. Nevertheless, a careful analysis of $\mathbb{E}\left[\exp \left(i \xi\left(Z_{s}-Z_{t}\right)\right)\right]$ via (1.3) yields (1.4). This is the approach in [20] where existence of the local time of the Rosenblatt process was first established. In this paper we show a considerably more involved bound on the Fourier transform which in turn will yield deeper results regarding the irregularity properties of the sample paths. The following is the key result of our paper:

Proposition 1.3. Let $n \in \mathbb{N}$ and $0 \leq \eta<\frac{1-H}{2 H}$. Then, for any times $0 \leq u<U$, the Rosenblatt process satisfies

$$
\int_{[u, U]^{n}} \int_{\mathbb{R}^{n}} \prod_{j=1}^{n}\left|\xi_{j}\right|^{\eta}\left|\mathbb{E} \exp \left(i \sum_{j=1}^{n} \xi_{j} Z_{t_{j}}\right)\right| d \xi d t \leq C^{n} n^{2 n H(1+\eta)}(U-u)^{(1-H(1+\eta)) n},
$$

where the constant $C>0$ depends only on $H$ and $\eta$.

Proposition 1.3 can be applied to obtain the following Hölder conditions on $L(x, B)$. 
Theorem 1.4. Let $\left(Z_{t}\right)_{t \geq 0}$ be a Rosenblatt process with $H \in\left(\frac{1}{2}, 1\right)$. The local time $(x, t) \mapsto L(x,[0, t])$ is almost surely jointly continuous and has finite moments. For a finite closed interval $I \subset(0, \infty)$, let $L^{*}(I)=\sup _{x \in \mathbb{R}} L(x, I)$. There exist constants $C_{1}$ and $C_{2}$ such that, almost surely,

$$
\limsup _{r \rightarrow 0} \frac{L^{*}([s-r, s+r])}{r^{1-H}\left(\log \log r^{-1}\right)^{2 H}} \leq C_{1},
$$

for any $s \in I$ and

$$
\limsup _{r \rightarrow 0} \sup _{s \in I} \frac{L^{*}([s-r, s+r])}{r^{1-H}\left(\log r^{-1}\right)^{2 H}} \leq C_{2} .
$$

In particular, the local time $L(x, I)$ is well defined for any fixed $x$ and interval $I \subset(0, \infty)$. Explicit estimates for the moments of the local time are provided in Theorem 3.1 below. As a direct corollary we obtain:

Corollary 1.5. For any finite closed interval $I \subset(0, \infty)$ there exists constants $C_{1}$ and $C_{2}$, independent of $x$ and $t$, such that, almost surely, for every $t \in I$ and every $x \in \mathbb{R}$

$$
\limsup _{r \rightarrow 0} \frac{L(x,[t-r, t+r])}{r^{1-H}\left(\log \log r^{-1}\right)^{2 H}} \leq C_{1},
$$

and for every $x \in \mathbb{R}$

$$
\limsup _{r \rightarrow 0} \sup _{t \in I} \frac{L(x,[t-r, t+r])}{r^{1-H}\left(\log r^{-1}\right)^{2 H}} \leq C_{2} .
$$

Moreover, we get the following for a particular Hausdorff measure:

Corollary 1.6. Let $I \subset(0, \infty)$ be a finite closed interval. There exists a constant $C$ such that for every $x \in \mathbb{R}$ we have

$$
\mathcal{H}_{\phi}\left(Z^{-1}(x) \cap I\right) \geq C L(x, I), \quad \text { a.s. }
$$

where $\mathcal{H}_{\phi}$ denotes $\phi$-Hausdorff measure with $\phi(r)=r^{1-H}\left(\log \log r^{-1}\right)^{2 H}$.

Furthermore, we can get a result on the behavior of the trajectories of $Z$.

Corollary 1.7. Let $I \subset(0, \infty)$ be a finite closed interval. There exists a constant $C>0$ such that for every $s \in I$ we have, almost surely,

$$
\liminf _{r \rightarrow 0} \sup _{s-r<t<s+r} \frac{\left|Z_{t}-Z_{s}\right|}{r^{H}\left(\log \log r^{-1}\right)^{-2 H}} \geq C,
$$

and

$$
\liminf _{r \rightarrow 0} \inf _{s \in I} \sup _{s-r<t<s+r} \frac{\left|Z_{t}-Z_{s}\right|}{r^{H}\left(\log \log r^{-1}\right)^{-2 H}} \geq C .
$$

In particular, $Z$ is almost surely nowhere differentiable. 
In Section 2 we establish our main result Proposition 1.3. As we are dealing with a second order Hermite process, we are forced to control from below singular values of somewhat unwieldy operators (see Remark 5.3 below). For this purpose we need to introduce several technical lemmas exhibiting tools from operator theory and harmonic analysis, including the theory of weighted integrals. Their proofs are postponed into Section 5. Section 3 is dedicated to some results regarding the existence and joint continuity of the local time. In particular, bounds on the moments of $L(x, B)$ are obtained. Finally, in Section 4, Theorem 1.4 and Corollaries 1.5 1.7 are established.

\section{Integrability of the Fourier transform}

The purpose of this section is to provide a proof of Proposition 1.3. We first outline the main steps as Lemmas 2.1 2.4 and then we establish (1.5). The proofs of the lemmas are carried out in Section 5 .

We use the following normalization for the Fourier transform

$$
\widehat{f}(\xi):=\int_{\mathbb{R}} e^{-i \xi x} f(x) d x,
$$

for $\xi \in \mathbb{R}$ and $f \in C_{0}^{\infty}(\mathbb{R})$. The norm in the weighted space $L_{G}^{2}$ is defined as $\|f\|_{L_{G}^{2}}^{2}:=\int_{\mathbb{R}}|f(x)|^{2} G(x) d x$.

First, we obtain a representation of the left-hand side of (1.5) using the eigenvalues of an integral operator.

Lemma 2.1. Let $t \in \mathbb{R}_{+}^{n}, \xi \in \mathbb{R}^{n}$, and let $A_{t, \xi}: L_{G}^{2}(\mathbb{R}) \rightarrow L_{G}^{2}(\mathbb{R})$, be the operator given by

$$
\left(A_{t, \xi} f\right)(x)=\int_{\mathbb{R}} \sum_{j=1}^{n} \xi_{j} \frac{e^{i t_{j}(x-y)}-1}{i(x-y)} f(y)|y|^{-H} d y
$$

Let $\left(\lambda_{k}\right)_{k \geq 1}$ be the sequence of the singular valued of $A_{t, \xi}$. Then,

$$
\left|\mathbb{E} \exp \left(i \sum_{j=1}^{n} \xi_{j} Z_{t_{j}}\right)\right|=\prod_{k \geq 1} \frac{1}{\left(1+4 \lambda_{k}^{2}\right)^{1 / 4}} .
$$

Next, instead of studying the properties of $\lambda_{k}$, defined as in Lemma 2.1, we introduce an operator that is unitarily equivalent to $A_{t, \xi}$, and obtain estimates for its singular values.

Lemma 2.2. The operator $A_{t, \xi}$ is unitarily equivalent to

$$
B_{t, \xi}:=c(H) K_{H / 2} M_{g} K_{H / 2}: L^{2}(\mathbb{R}) \rightarrow L^{2}(\mathbb{R})
$$

\footnotetext{
${ }^{2}$ Note that for a self-adjoint operator $T$ the singular value sequence consists of absolute values of the eigenvalues, repeated according to multiplicity. For later purposes it is useful to speak of singular values since some of the results we will be using in Section 5.1 are valid only for singular values.
} 
where $g(x)=\sum_{j=1}^{n} \xi_{j} \chi_{\left[0, t_{j}\right]}(x), M_{g}$ is the multiplication operator $\left(M_{g} f\right)(x):=g(x) f(x)$ and $K_{\alpha}$ is a convolution operator defined via the Fourier transform:

$$
\left(\widehat{K_{\alpha} f}\right)(x):=|x|^{-\alpha} \widehat{f}(x),
$$

for $\alpha \in(-1 / 2,1 / 2)$. Furthermore, if $t_{0}=0<t_{1}<\cdots<t_{n} \leq 1$, and we set $\xi_{0}=0$, the $n$th singular value $\mu_{n}$ of $B_{t, \xi}$ (and then also of $A_{t, \xi}$ ) satisfies

$$
\mu_{n}\left(B_{t, \xi}\right) \geq C(H)\left(\max _{1 \leq j \leq n}\left|\xi_{j}-\xi_{j-1}\right|\left|t_{j}-t_{j-1}\right|^{H}\right) \tilde{\mu}_{n}^{2},
$$

where $\tilde{\mu}_{n} \sim c(H) n^{-H / 2}$, and $c(H), C(H)>0$ are constants that only depends on $H$.

The next step is to provide bounds on integrals involving expressions like on the right-hand side of (2.2). First, the following result holds.

Lemma 2.3. Define for $s \in \mathbb{R}$, the function

$$
G(s):=\prod_{k=1}^{\infty}\left(1+4 s^{2} \tilde{\mu}_{k}^{4}\right)^{-1 / 4} .
$$

Then the product (2.3) converges and $G(s)>0$ for $s>0$. Moreover, there is a constants $c_{3}=C_{3}(H)$ such that, for all $\beta \geq 1$,

$$
\int_{0}^{\infty} s^{\beta-1} G(s) d s \leq c_{3}^{\beta H} \Gamma(\beta H),
$$

where $\Gamma$ is the Gamma function.

The next technical result gives an expression for an integral of a function similar to the maximum term appearing in (2.2). In particular, let $f_{0}: \mathbb{R}_{+}^{n} \times \mathbb{R}^{n} \rightarrow \mathbb{R}_{+}$be given by

$$
f_{0}(t, y):=t_{1}^{H}\left|y_{1}\right| \vee t_{2}^{H}\left|y_{2}\right| \vee \cdots \vee t_{n}^{H}\left|y_{n}\right|
$$

Then the following holds:

Lemma 2.4. Assume that $\gamma_{j} \in\left[0, H^{-1}-1\right)$ for each $j$ where $H \in(0,1)$, and write $\gamma_{a v}=n^{-1} \sum_{j=1}^{n} \gamma_{j}$. Then,

$$
\begin{gathered}
\int_{S^{n-1}} \int_{\substack{t_{1}+\cdots+t_{n} \leq 1 \\
t_{1}, \ldots, t_{n} \geq 0}} \prod_{j=1}^{n}\left|y_{j}\right|^{\gamma_{j}}\left(f_{0}(t, y)\right)^{-n\left(1+\gamma_{a v}\right)} d t \mathcal{H}^{n-1}(d y) \\
=\frac{n^{1 / 2}\left(1+\gamma_{a v}\right) \prod_{j=1}^{n}\left[\frac{2}{1+\gamma_{j}} \Gamma\left(1-H\left(1+\gamma_{j}\right)\right)\right]}{\Gamma\left(n\left(1-H\left(1+\gamma_{a v}\right)\right)+1\right)}
\end{gathered}
$$

where $\mathcal{H}^{n-1}(d y)$ is the $(n-1)$-dimensional Hausdorff measure.

Now that the main technical steps are outlined, we present the proof of Proposition 1.3 . 
Proof of Proposition 1.3. Note first that, by Lemma 2.1.

$$
\begin{aligned}
& \int_{[u, U]^{n}} \int_{\mathbb{R}^{n}} \prod_{j=1}^{n}\left|\xi_{j}\right|^{\eta}\left|\mathbb{E} \exp \left(i \sum_{j=1}^{n} \xi_{j} Z_{t_{j}}\right)\right| d \xi d t \\
= & \left.\int_{t \in[u, U]^{n}} \int_{\xi \in \mathbb{R}^{n}} \prod_{j=1}^{n}\left|\xi_{j}\right|^{\eta} \prod_{k=1}^{\infty}\left(1+4 \mu_{k}\left(A_{t, \xi}\right)^{2}\right)\right)^{-1 / 4} d \xi d t=: \quad I .
\end{aligned}
$$

We first perform a reduction to the case $u=0$. For that purpose recall that Lemma 2.2 verifies that the operator $A_{t, \xi}$ is unitarily equivalent to the operator $c(H / 2) \sum_{j=1}^{n} \xi_{j} K_{H / 2} M_{\chi_{\left[0, t_{j}\right]}} K_{H / 2}$. Next, assuming that $t_{j} \geq u$ for all $j \in\{1, \ldots, n\}$, Lemma 5.1 yields that, for every $k \geq 1$,

$$
\begin{aligned}
& \mu_{k}\left(\sum_{j=1}^{n} \xi_{j} K_{H / 2} M_{\chi_{\left[u, t_{j}\right]}} K_{H / 2}\right)=\mu_{k}\left(U_{H / 2,[u, \infty)} \sum_{j=1}^{n} \xi_{j} K_{H / 2} M_{\chi_{\left[0, t_{j}\right]}} K_{H / 2}\right) \\
\leq & R \mu_{k}\left(A_{t, \xi}\right),
\end{aligned}
$$

where we used the Minimax principle (see [4, Corollary III.1.2]) and $R:=\left\|U_{H / 2,[u, \infty)}\right\|$. On the other hand, by the translation invariance of the Fourier-multipliers $K_{\alpha}$ we see that, for every $k \geq 1$,

$$
\mu_{k}\left(\sum_{j=1}^{n} \xi_{j} K_{H / 2} M_{\chi_{\left[u, t_{j}\right]}} K_{H / 2}\right)=\mu_{k}\left(\sum_{j=1}^{n} \xi_{j} K_{H / 2} M_{\chi_{\left[0, t_{j}-u\right]}} K_{H / 2}\right) .
$$

Then, $\mu_{k}\left(A_{t, \xi}\right) \geq R^{-1} \mu_{k}\left(A_{t-u e, \xi}\right)$, where $e=(1, \ldots, 1)$, and hence a change of variables in the integral $I$ yields

$$
\left.I \leq \int_{t \in[U-u]^{n}} \int_{\xi \in \mathbb{R}^{n}} \prod_{j=1}^{n}\left|\xi_{j}\right|^{\eta} \prod_{k=1}^{\infty}\left(1+4 A^{-1} \mu_{k}\left(A_{t, \xi}\right)^{2}\right)\right)^{-1 / 4} d \xi d t:=I^{\prime}
$$

Above, $I^{\prime}$ is our integral $I$ reduced to the case $u=0$ up to a constant $R$ in the integrand.

We then assume that $u=0$ and consider the integral $I^{\prime}$. By symmetry, $I^{\prime}$ equals $n$ ! times the same integral restricted to the ordered set $\left\{0 \leq t_{1}<\cdots<t_{n} \leq U-u\right\}$. Again, $A_{t, \xi}$ is unitarily equivalent to $K_{H / 2} M_{g} K_{H / 2}$, where

$$
g=\sum_{j=1}^{n} \xi_{j} \chi_{\left[0, t_{j}\right]}=\sum_{j=1}^{n} \xi_{j}^{\prime} \chi_{I_{j}}
$$

where $I_{j}=\left[t_{j-1}, t_{j}\right]$ with $t_{0}:=0$ and $\xi_{j}^{\prime}:=\sum_{\ell=j}^{n} \xi_{j}, j=1, \ldots, n$. For notational purposes we set $\xi_{n+1}^{\prime}=0$ We also make the change of variables $t_{j}^{\prime}=t_{j}-t_{j-1}$, $j=1, \ldots, n$. Both changes of variables have Jacobian equal to 1 . According to (2.2) of Lemma 2.2 and Lemma 2.3 .

$$
I^{\prime} \leq n ! \int_{\substack{t_{1}^{\prime}+\ldots+t_{n}^{\prime} \leq U \\ t_{1}^{\prime}, \ldots, t_{n}^{\prime}>0}} \int_{\xi^{\prime} \in \mathbb{R}^{n}} \prod_{j=1}^{n}\left|\xi_{j}^{\prime}-\xi_{j+1}^{\prime}\right|^{\eta} G\left(c^{\prime} f_{0}\left(t^{\prime}, \xi^{\prime}\right)\right) d t^{\prime} d \xi^{\prime}
$$


where $c^{\prime}>0$ (which also incorporates the constant $R$ ) and $f_{0}$ is as in (2.5).

Next, note that for every $\eta \geq 0,\left|\xi_{j}^{\prime}-\xi_{j+1}^{\prime}\right|^{\eta} \leq 2^{(\eta-1) \vee 0}\left(\left|\xi_{j}^{\prime}\right|^{\eta}+\left|\xi_{j+1}^{\prime}\right|^{\eta}\right)$ and thus

$$
\prod_{j=1}^{n}\left|\xi_{j}^{\prime}-\xi_{j+1}^{\prime}\right|^{\eta} \leq c^{\prime \prime n} \sum \prod\left|\xi_{j}^{\prime}\right|^{\gamma_{j}}
$$

where $c^{\prime \prime}:=2^{(\eta-1) \vee 0}$ and the exponents in each term of the sum satisfy $\gamma_{j} \in\{0, \eta, 2 \eta\}$, and $\gamma_{\mathrm{av}}:=n^{-1} \sum_{j=1}^{n} \gamma_{j}=\eta$. The number of summands is $2^{n-1}$, since $\xi_{n+1}^{\prime}=0$. For any fixed exponent sequence $\left(\gamma_{1}, \ldots, \gamma_{n}\right)$ we switch to polar coordinates $\left|\xi^{\prime}\right|=r^{\prime}$, $\xi^{\prime} / r=w^{\prime}$ :

$$
\begin{aligned}
& \int_{\substack{t_{1}^{\prime}+\ldots+t_{n}^{\prime} \leq U \\
t_{1}^{\prime}, \ldots, t_{n}^{\prime}>0}} \int_{\xi^{\prime} \in \mathbb{R}^{n}} \prod_{j=1}^{n}\left|\xi_{j}^{\prime}\right|^{\gamma_{j}} G\left(c^{\prime} f_{0}\left(t^{\prime}, \xi^{\prime}\right)\right) d t^{\prime} d \xi^{\prime} \\
= & \int_{\substack{t_{1}^{\prime}+\ldots+t_{n}^{\prime} \leq U \\
t_{1}^{\prime}, \ldots, t_{n}^{\prime}>0}} \int_{\left|w^{\prime}\right|=1} \int_{0}^{\infty} r^{\prime n-1} r^{\prime n \eta} \prod_{j=1}^{n}\left|w_{j}^{\prime}\right|^{\gamma_{j}} G\left(c^{\prime} r^{\prime} f_{0}\left(t^{\prime}, w^{\prime}\right)\right) d r^{\prime} \mathcal{H}^{n-1}\left(d w^{\prime}\right) d t^{\prime} \\
= & \left(c^{\prime}\right)^{-n(1+\eta)}\left(\int_{0}^{\infty} R^{n(1+\eta)-1} G(R) d R\right) \times \\
& \times \int_{\substack{t_{1}^{\prime}+\ldots+t_{n}^{\prime} \leq U \\
t_{1}^{\prime}, \ldots, t_{n}^{\prime}>0}} \int_{\left|w^{\prime}\right|=1} \prod_{j=1}^{n}\left|w_{j}^{\prime}\right|^{\gamma_{j}}\left(f_{0}\left(t^{\prime}, w^{\prime}\right)\right)^{-n(1+\eta)} \mathcal{H}^{n-1}\left(d w^{\prime}\right) d t^{\prime},
\end{aligned}
$$

where $R:=c^{\prime} r^{\prime} f_{0}\left(t^{\prime}, w^{\prime}\right)$. Apply Lemma 2.3 to estimate the $R$-integral and set $t^{\prime}=U v$. By the $H$-homogeneity of $f_{0}$ in the $t$-variable the previous integral is upper bounded by

$$
\begin{aligned}
c_{3}^{n(1+\eta) H}\left(c^{\prime}\right)^{-n(1+\eta)} \Gamma(n(1+\eta) H) \times & \\
& \times U^{(1-H(1+\eta)) n} \int_{\substack{v_{1}+\ldots+v_{n} \leq 1 \\
v_{1}, \ldots, v_{n}>0}} \int_{\left|w^{\prime}\right|=1} \prod_{j=1}^{n}\left|w_{j}^{\prime}\right|^{\gamma_{j}}\left(f_{0}\left(v, w^{\prime}\right)\right)^{-n(1+\eta)} \mathcal{H}^{n-1}(d w) d v
\end{aligned}
$$

for some constant $c_{3}=C_{3}(H)>0$. Next, by Lemma 2.4 the above is further bounded by

$$
\begin{aligned}
& C(H, n) U^{(1-H(1+\eta)) n} \Gamma(n(1+\eta) H) \frac{n^{1 / 2}(1+\eta) \prod_{j=1}^{n}\left[\frac{2}{1+\gamma_{j}} \Gamma\left(1-H\left(1+\gamma_{j}\right)\right)\right]}{\left(c^{\prime}\right)^{n(1+\eta)} \Gamma(n(1-H(1+\eta))+1)} \\
\leq & C(H, n) U^{(1-H(1+\eta)) n}\left(\frac{2}{c^{1+\eta}} \Gamma(1-H(1+2 \eta))\right)^{n} \frac{\Gamma(n H(1+\eta)+1)}{n^{1 / 2} \Gamma(n(1-H(1+\eta))+1)},
\end{aligned}
$$

where $C(H, n):=\left(c_{3} / c^{\prime}\right)^{-n(1+\eta) H}$ and we used the fact that the Gamma function is decreasing on $(0,1)$ and $\gamma_{j} \leq 2 \eta$. Summing over the $2^{n-1}$ different exponent sequences $\left(\gamma_{1}, \ldots, \gamma_{n}\right)$ and recalling the $n$ ! factor introduced in the beginning of the 
proof yields

$$
\begin{aligned}
I & \leq C(H) U^{(1-H(1+\eta)) n}\left(\frac{4}{c^{\prime 1+\eta}} \Gamma(1-H(1+2 \eta))\right)^{n} \frac{\Gamma(n+1) \Gamma(n H(1+\eta)+1)}{\Gamma(n(1-H(1+\eta))+1)} \\
& =U^{(1-H(1+\eta)) n} C^{n} \frac{\Gamma(n+1) \Gamma(n H(1+\eta)+1)}{\Gamma(n(1-H(1+\eta))+1)},
\end{aligned}
$$

where $C>0$ depends only on $H$ and $\eta$. Finally, an application of Stirling's formula establishes (1.5):

$$
I \leq C^{n} n^{2 n H(1+\eta)} U^{(1-H(1+\eta)) n},
$$

for a different $C>0$ depending only on $H$ and $\eta$ (recall that we reduced to the case $u=0)$.

Proposition 1.3 establishes integrability properties of the Fourier transform of the local time and leads to good moment estimates for the local time in next section. In turn, Section 4 uses the moment estimates to deduce several important results regarding the asymptotic behavior of the local time.

\section{Joint continuity of the local times and moment estimates}

In the present section we apply Proposition 1.3 to produce moment estimates for the local time, that are of some independent interest.

Let $t>0$ and $x \in \mathbb{R}$. We recall from [20] that the local time $L(x, t):=L(x,[0, t])$ for the Rosenblatt process $Z$ exists and admits the representation ${ }^{3}$

$$
L(x, t)=\frac{1}{2 \pi} \int_{\mathbb{R}} \int_{0}^{t} e^{i \xi\left(x-Z_{s}\right)} d s d \xi .
$$

Our next step is to show that $L(x, t)$ is Hölder-continuous both in time and space, and also to establish bounds on its moments.

Theorem 3.1. For every $0 \leq s<t$ and $x \in \mathbb{R}$,

$$
\mathbb{E}|L(x, t)-L(x, s)|^{n} \leq c^{n} n^{n 2 H}|t-s|^{(1-H) n} .
$$

Moreover, for any $0 \leq \gamma<\frac{1-H}{2 H}$ and $y \in \mathbb{R}$, we have

$$
\left|\mathbb{E}(L(x+y,[s, t])-L(x,[s, t]))^{n}\right| \leq c^{n} n^{n 2 H(1+\gamma)}|t-s|^{(1-H-\gamma H) n}|y|^{\gamma n} .
$$

In both inequalities the constant $c$ depends only on $\gamma$ and $H$.

\footnotetext{
${ }^{3} \mathrm{~A}$ priori, after [20, the occupation density is defined only as an $L^{2}$-density, and hence they are not necessarily well-defined for fixed $x$. Thus some of our computations below might seem unfounded. However, one may use Proposition 1.3 to first prove uniform regularity bounds for suitable mollifications, which justifies (3.1) for any fixed $x$ and our computations later on.
} 
Proof. First, by (3.1),

$$
\begin{aligned}
& \mathbb{E}(L(x+y,[s, t])-L(x,[s, t]))^{n} \\
= & (2 \pi)^{-n} \int_{\mathbb{R}^{n}} \int_{[s, t]^{n}}\left(\prod_{j=1}^{n}\left(\exp \left(i \xi_{j}(x+y)\right)-\exp \left(i \xi_{j} x\right)\right)\right) \mathbb{E} \exp \left(-i \sum_{j=1}^{n} \xi_{j} Z_{v_{j}}\right) d v d \xi
\end{aligned}
$$

Next, since $\gamma \in[0,1)$ (recall that $H>1 / 2$ ), we have

$$
\prod_{j=1}^{n}\left|\exp \left(i \xi_{j} y\right)-1\right| \leq 2^{n} \prod_{j=1}^{n}\left(|y|\left|\xi_{j}\right| \wedge 1\right) \leq 2^{n} \prod_{j=1}^{n}\left(\left(|y|\left|\xi_{j}\right|\right)^{\gamma} \wedge 1\right) \leq 2^{n}|y|^{\gamma n} \prod_{j=1}^{n}\left|\xi_{j}\right|^{\gamma},
$$

where we have used that $\left|e^{i x}-1\right| \leq|x| \wedge 2 \leq 2(|x| \wedge 1)$, for all $x$. Therefore,

$$
\begin{aligned}
& \left|\mathbb{E}(L(x+y,[s, t])-L(x,[s, t]))^{n}\right| \\
\leq & \pi^{-n}|y|^{\gamma n} \int_{\mathbb{R}^{n}} \int_{[s, t]^{n}} \prod_{j=1}^{n}\left|\xi_{j}\right|^{\gamma}\left|\mathbb{E} \exp \left(-i \sum_{j=1}^{n} \xi_{j} Z_{v_{j}}\right)\right| d v d \xi .
\end{aligned}
$$

Now, Proposition 1.3 with $\eta=\gamma$ yields:

$$
\left|\mathbb{E}(L(x+y,[s, t])-L(x,[s, t]))^{n}\right| \leq C^{n}|y|^{\gamma n}(t-s)^{1-H(1+\gamma) n} n^{2 n H(1+\gamma)},
$$

where $C>0$ is a function of $H$ and $\gamma$ and (3.3) is established.

Similarly, by (3.1), using $L(x, s) \leq L(x, t)$ for $0 \leq s<t$,

$$
\begin{aligned}
& \mathbb{E}|L(x, t)-L(x, s)|^{n} \\
= & \left((2 \pi)^{-n} \int_{[s, t]^{n}} \int_{\mathbb{R}^{n}} \exp \left(i x \sum_{j=1}^{n} \xi_{j}\right) \mathbb{E} \exp \left(-i \sum_{j=1}^{n} \xi_{j} Z_{u_{j}}\right) d \xi d u\right) \\
\leq & (2 \pi)^{-n} \int_{[s, t]^{n}} \int_{\mathbb{R}^{n}}\left|\mathbb{E} \exp \left(-i \sum_{j=1}^{n} \xi_{j} Z_{u_{j}}\right)\right| d \xi d u \\
\leq & \left(\frac{C}{2 \pi}\right)^{n} n^{2 n H}(t-s)^{(1-H) n},
\end{aligned}
$$

where the last inequality follows from Proposition 1.3 with $\eta=0$, and $C>0$ is a function of $H$ and $\gamma$.

As an immediate consequence of the above moment bounds and Kolmogorov criterion (see e.g. [15, Theorem 3.23]) we obtain:

Corollary 3.2. Almost surely, the local time $L(x, t)$ is jointly Hölder continuous in $t$ and $x$.

The next theorem is a modification of Theorem 3.1, where one shifts the process in the $x$-direction by the value $Z_{a}$, where $a$ is a fixed point. 
Theorem 3.3. Let $s<t$ and let $a>0$ satisfy $a \leq s$ or $a \geq t$. Then,

$$
\mathbb{E}\left|L\left(x+Z_{a}, t\right)-L\left(x+Z_{a}, s\right)\right|^{n} \leq c^{n}(n)^{n 2 H}|t-s|^{(1-H) n} .
$$

Moreover, for any $0 \leq \gamma<\left(H^{-1}-1\right) / 2$,

$$
\left|\mathbb{E}\left(L\left(x+y+Z_{a},[s, t]\right)-L\left(x+Z_{a},[s, t]\right)\right)^{n}\right| \leq c^{n}(n)^{n 2 H(1+\gamma)}|t-s|^{(1-H-\gamma H) n}|y|^{\gamma n} .
$$

In both cases the constant $c>0$ depends only on $\gamma$ and $H$.

Proof. Let $Y_{t}=Z_{t}-Z_{a}$. The occupation measure of $Y$ is just the occupation measure of $Z_{t}$ translated by the (random) constant $Z_{a}$. Since the occupation measure of $Z_{t}$ has a continuous density, the occupation measure of $Y_{t}$ has also a continuous density given by $L_{Y}(t, x)=L_{Z}\left(t, x+Z_{a}\right)$. Thus, in order to prove the claim, it suffices to show the estimates for $L_{Y}(t, x)$. For the first claim, we then proceed as before, noting that, again, $L_{Y}(x, s) \leq L_{Y}(x, t)$,

$$
\begin{aligned}
& \mathbb{E}\left|L_{Y}(x, t)-L_{Y}(x, s)\right|^{n} \\
= & (2 \pi)^{-n} \int_{[s, t]^{n}} \int_{\mathbb{R}^{n}} \exp \left(i x \sum_{j=1}^{n} \xi_{j}\right) \mathbb{E} \exp \left(i \sum_{j=1}^{n} \xi_{j} Y_{u_{j}}\right) d \xi d u \\
\leq & (2 \pi)^{-n} \int_{[s, t]^{n}} \int_{\mathbb{R}^{n}}\left|\mathbb{E} \exp \left(i \sum_{j=1}^{n} y_{j} Y_{u_{j}}\right)\right| d y d u .
\end{aligned}
$$

Let first $a \leq s$. By stationarity of the increments, we have

$$
\mathbb{E} \exp \left(i \sum_{j=1}^{n} y_{j} Y_{u_{j}}\right)=\mathbb{E} \exp \left(i \sum_{j=1}^{n} y_{j}\left(Z_{u_{j}}-Z_{a}\right)\right)=\mathbb{E} \exp \left(i \sum_{j=1}^{n} y_{j} Z_{u_{j}-a}\right) .
$$

Thus change of variable $v_{j}=u_{j}-a$ gives

$$
\int_{[s, t]^{n}} \int_{\mathbb{R}^{n}}\left|\mathbb{E} \exp \left(i \sum_{j=1}^{n} y_{j} Y_{u_{j}}\right)\right| d y d u=\int_{[s-a, t-a]^{n}} \int_{\mathbb{R}^{n}}\left|\mathbb{E} \exp \left(i \sum_{j=1}^{n} y_{j} Z_{v_{j}}\right)\right| d y d v
$$

from which the claim follows by Proposition 1.3 just as in the proof of Theorem 3.1 with $\eta=0$, and $C>0$ is a function of $H$ and $\gamma$. Similarly, for $a \geq t$ stationarity of increments imply

$$
\mathbb{E} \exp \left(i \sum_{j=1}^{n} y_{j} Y_{u_{j}}\right)=\mathbb{E} \exp \left(-i \sum_{j=1}^{n} y_{j}\left(Z_{a}-Z_{u_{j}}\right)\right)=\mathbb{E} \exp \left(-i \sum_{j=1}^{n} y_{j} Z_{a-u_{j}}\right) .
$$

This can be treated exactly the same way by using first change of variable $v_{j}=a-u_{j}$. This concludes the proof.

The moment bounds obtained above translate into the following tail estimates. Their proof is a standard application of Chebychev's inequality, and hence we omit the proof. 
Corollary 3.4. (i) For any finite closed interval $I \subset(0, \infty)$,

$$
\mathbb{P}\left(L(x, I) \geq|I|^{1-H} u^{2 H}\right) \leq C_{1} \exp \left(-c_{1} u\right)
$$

and

$$
\mathbb{P}\left(|L(x, I)-L(y, I)| \geq|I|^{1-H-\gamma H}|x-y|^{\gamma} u^{2 H(1+\gamma)}\right) \leq C_{2} \exp \left(-c_{2} u\right) .
$$

(ii) For $I=[a, a+r]$ or $I=[a-r, a]$, we have

$$
\mathbb{P}\left(L\left(x+Z_{a}, I\right) \geq r^{1-H} u^{2 H}\right) \leq C_{1} \exp \left(-c_{1} u\right)
$$

and

$$
\mathbb{P}\left(\left|L\left(x+Z_{a}, I\right)-L\left(y+Z_{a}, I\right)\right| \geq r^{1-H-\gamma H}|x-y|^{\gamma} u^{2 H(1+\gamma)}\right) \leq C_{2} \exp \left(-c_{2} u\right) .
$$

\section{Proofs of main theorems}

In this section we present proofs to our main results, namely Theorem 1.4 and Corollaries 1.5.1.7. We start with two auxiliary lemmas.

Lemma 4.1. There exists $\eta>0$ such that

$$
\mathbb{E} e^{\eta\left|Z_{1}\right|}<\infty
$$

Proof. This follows by observing that by Lemma 2.1 the characteristic function of $Z_{1}$ has a bounded analytic extension to a strip $\left\{|\operatorname{Im} \theta|<\delta_{0}\right\}$ for some $\delta_{0}>0$.

Proposition 4.2. Let $\left(Z_{t}\right)_{t \geq 0}$ be the Rosenblatt process and set $I=[s-h, s+h]$, where $h \leq 1$ and $s>0$. Then,

$$
\mathbb{P}\left(\sup _{t \in I}\left|Z_{t}-Z_{s}\right| \geq u\right) \leq C \exp \left(-\frac{u}{c_{1} h^{H}}\right)
$$

where $c_{1}$ and $C$ are constants that depend only on $H$.

Proof. By stationarity of increments and self-similarity we have the distributional equality $\left(\left|Z_{r}-Z_{v}\right|\right)_{r \in I} \sim\left(|h|^{H}\left|Z_{r}\right|\right)_{r \in[0,1]}$. Hence it is enough to consider case $s=0$ and $h=1$. At this point we recall the Garsia-Rodemich-Rumsey inequality [10].

Proposition 4.3. Let $\Psi(u)$ be a non-negative even function on $(-\infty, \infty)$ and $p(u)$ be a non-negative even function on $[-1,1]$. Assume both $p(u)$ and $\Psi(u)$ are non decreasing for $u \geq 0$. Let $f(x)$ be continuous on $[0,1]$ and suppose that

$$
\int_{0}^{1} \int_{0}^{1} \Psi\left(\frac{f(x)-f(y)}{p(x-y)}\right) d x d y \leq B<\infty .
$$

Then, for all $s, t \in[0,1]$,

$$
|f(s)-f(t)| \leq 8 \int_{0}^{|s-t|} \Psi^{-1}\left(\frac{4 B}{u^{2}}\right) d p(u) .
$$


In particular 4 , if $\Psi(u)=|u|^{p}$ and $p(u)=|u|^{\alpha+1 / p}$ where $\alpha \geq 1 / p$ and $p \geq 1$, then for any continuous $f$ and $t \in[0,1]$,

$$
|f(t)-f(0)|^{p} \leq C_{\alpha, p} t^{\alpha p-1} \int_{[0,1]^{2}}|f(r)-f(v)|^{p}|r-v|^{-\alpha p-1} d r d v .
$$

Here the constant $C_{\alpha, p}$ is given by $C_{\alpha, p}=4 \cdot 8^{p}\left(\alpha+p^{-1}\right)^{p}\left(\alpha-p^{-1}\right)^{-p}$. Thus, for fixed $\alpha$ and large enough $p$, we have $C_{\alpha, p} \leq \tilde{C}^{p}$, where $\tilde{C}$ depends on the chosen $\alpha$. We apply this to $f(t)=Z_{t}$ and choose $\alpha=\frac{H}{2}$, in order to obtain for $p>4 / H$ :

$$
\sup _{t \in[0,1]}\left|Z_{t}\right|^{p} \leq C^{\prime p} \int_{[0,1]^{2}}\left|Z_{r}-Z_{v}\right|^{p}|r-v|^{-H p / 2-1} d r d v \text {. }
$$

By stationarity of increments and self-similarity we have $\mathbb{E}\left|Z_{r}-Z_{v}\right|^{p}=|r-v|^{H p} \mathbb{E}\left|Z_{1}\right|^{p}$, which leads to

$$
\begin{aligned}
\mathbb{E} \sup _{t \in[0,1]}\left|Z_{t}\right|^{p} & \leq C^{\prime p} \mathbb{E}\left|Z_{1}\right|^{p} \int_{[0,1]^{2}}|r-v|^{H p / 2-1} d r d v \leq c_{2} C^{\prime p} \mathbb{E}\left|Z_{1}\right|^{p} \\
& \leq C^{p} \mathbb{E}\left|Z_{1}\right|^{p}
\end{aligned}
$$

By Lemma 4.1 we may choose $\eta>0$ such that $\mathbb{E} e^{\eta\left|Z_{1}\right|}<\infty$. Then we have

$$
\begin{aligned}
\mathbb{E} e^{\eta C^{-1} \sup _{t \in[0,1]}\left|Z_{t}\right|} & =\sum_{k=0}^{\infty} \frac{\left(\eta C^{-1}\right)^{k} \mathbb{E} \sup _{t \in[0,1]}\left|Z_{t}\right|^{k}}{k !} \leq \sum_{k=0}^{\infty} \frac{\eta^{k} \mathbb{E}\left|Z_{1}\right|^{k}}{k !} \\
& =\mathbb{E} \sum_{k=0}^{\infty} \frac{\eta^{k}\left|Z_{1}\right|^{k}}{k !}=\mathbb{E} e^{\eta\left|Z_{1}\right|}<\infty
\end{aligned}
$$

The claim follows easily from this by Chebyshev's inequality.

We are now in position to prove Theorem 1.4. After we have the moment and tail estimates from Section 3 at our disposal, the remaining ideas of the proof follow closely those of [2, Theorem 4.3]. The main difference is that in our case, we do not have Gaussian structures at our disposal leading to some modifications.

Proof of Theorem 1.4. We divide the proof into five steps. In the first four steps we prove (1.6). The proof of (1.7) will then be established in step 5 .

Throughout steps 1 -4 we denote $g(r)=r^{1-H}\left(\log \log r^{-1}\right)^{2 H}$, where $r<e$, and $C_{n}^{+}:=\left[s, s+2^{-n}\right], C_{n}^{-}:=\left[s-2^{-n}, s\right]$. It actually suffices to prove

$$
\limsup _{n \rightarrow \infty} \frac{L^{*}\left(C_{n}^{ \pm}\right)}{g\left(2^{-n}\right)} \leq C
$$

almost surely. Moreover, it suffices to consider only the interval $C_{n}^{+}$, as $C_{n}^{-}$can be treated by exactly the same arguments and by considering the interval $[a-r, a]$ in Corollary 3.4. Throughout the proof, we denote by $c_{i}, i=1,2, \ldots$ generic constants

\footnotetext{
${ }^{4}$ Actually, this special case can also be obtained from a standard trace theorem for Besov spaces.
} 
that varies throughout the proof. We also write simply $C_{n}=\left[s, s+2^{-n}\right]$ instead of $C_{n}^{+}$.

Step 1: Set $u=2 c_{1} 2^{-n H} \log n$, where $c_{1}$ is given in Proposition 4.2. Then Proposition 4.2 gives

$$
\mathbb{P}\left(\sup _{t \in C_{n}}\left|Z_{t}-Z_{s}\right| \geq 2 c_{1} 2^{-n H} \log n\right) \leq c_{2} \exp (-2 \log n)=c_{2} n^{-2} .
$$

Hence Borel-Cantelli lemma implies that there exists $n_{1}=n_{1}(\omega)$ such that for $n \geq n_{1}$ we have

$$
\sup _{t \in C_{n}}\left|Z_{t}-Z_{s}\right| \leq 2 c_{1} 2^{-n H} \log n .
$$

Step 2: Set $\theta_{n}=2^{-n H}\left(\log \log 2^{n}\right)^{-2 H}$ and define

$$
G_{n}=\left\{x \in \mathbb{R}:|x| \leq 2 c_{1} 2^{-n H} \log n, x=\theta_{n} p, \text { for some } p \in \mathbb{Z}\right\} .
$$

Then

$$
\# G_{n} \leq c_{3}(\log n)^{1+2 H}
$$

and (3.8) implies

$$
\mathbb{P}\left(\max _{x \in G_{n}} L\left(x+Z_{s}, C_{n}\right) \geq c_{4} g\left(2^{-n}\right)\right) \leq c_{5}(\log n)^{1+2 H} n^{-c_{6} b_{1}}
$$

which is summable by choosing $c_{4}$ large enough which in turn forces $c_{6}$ large. Thus Borel-Cantelli gives for large enough $n \geq n_{2}(\omega) \geq n_{1}(\omega)$ that

$$
\left.\max _{x \in G_{n}} L\left(x+Z_{s}, C_{n}\right) \leq c_{4} g\left(2^{-n}\right)\right) .
$$

Step 3: Given integers $n, k \geq 1$ and $x \in G_{n}$ we set

$$
F(n, k, x)=\left\{y=x+\theta_{n} \sum_{j=1}^{k} \varepsilon_{j} 2^{-j}: \varepsilon_{j} \in\{0,1\}, 1 \leq j \leq k\right\} .
$$

Pair of points $y_{1}, y_{2} \in F(n, k, x)$ is said to be linked if $y_{2}-y_{1}=\theta_{n} \varepsilon 2^{-k}$ for $\varepsilon \in\{0,1\}$. Next fix $0<\gamma<\frac{\frac{1}{H}-1}{2}$ and choose positive $\delta$ such that $\delta 2 H(1+\gamma)<\gamma$. Set

$$
B_{n}=\bigcup_{x \in G_{n}} \bigcup_{k=1}^{\infty} \bigcup_{y_{1}, y_{2}}\left\{\left|L\left(y_{1}+Z_{s}, C_{n}\right)-L\left(y_{2}+Z_{s}, C_{n}\right)\right| \geq 2^{-n(1-H-\gamma H)}\left|y_{1}-y_{2}\right|^{\gamma}\left(c_{7} 2^{\delta k} \log n\right)^{2 H(1+\gamma)}\right\}
$$

where $\cup_{y_{1}, y_{2}}$ is the union over all linked pairs $y_{1}, y_{2} \in F(n, k, x)$. Now (3.9) with $u=c_{7} 2^{\delta k} \log n$ implies

$$
\mathbb{P}\left(B_{n}\right) \leq c_{3}(\log n)^{1+2 H} \sum_{k=1}^{\infty} 4^{k} \exp \left(-c_{8} 2^{\delta k} \log n\right) .
$$

Here we have used the fact $\# G_{n} \leq C(\log n)^{1+2 H}$ and that for given $k$ there exists less than $4^{k}$ linked pairs $y_{1}, y_{2}$. Now, again by choosing $c_{7}$ large enough which makes 
$c_{8}$ large, we get

$$
\sum_{n=2}^{\infty}(\log n)^{1+2 H} \sum_{k=1}^{\infty} 4^{k} \exp \left(-c_{8} 2^{\delta k} \log n\right)<\infty .
$$

This further implies, again by Borel-Cantelli lemma, that $B_{n}$ occurs only finitely many times.

Step 4: Let $n$ be a fixed and assume that $y \in \mathbb{R}$ satisfies $|y| \leq 2 c_{1} 2^{-n H} \log n$. Then we may represent $y$ as $y=\lim _{k \rightarrow \infty} y_{k}$ with

$$
y_{k}=x+\theta_{n} \sum_{j=1}^{k} \varepsilon_{j} 2^{-j},
$$

where $y_{0}=x \in G_{n}$ and $\varepsilon_{j} \in\{0,1\}$. On the event $B_{n}^{c}$ we have

$$
\begin{aligned}
\left|L\left(x+Z_{s}, C_{n}\right)-L\left(y+Z_{s}, C_{n}\right)\right| & \leq \sum_{k=1}^{\infty}\left|L\left(y_{k}+Z_{s}, C_{n}\right)-L\left(y_{k-1}+Z_{s}, C_{n}\right)\right| \\
& \leq \sum_{k=1}^{\infty} 2^{-n(1-H-\gamma H)}\left|y_{k}-y_{k-1}\right|^{\gamma}\left(c_{7} 2^{\delta k} \log n\right)^{2 H(1+\gamma)} \\
& \leq \sum_{k=1}^{\infty} 2^{-n(1-H-\gamma H)} \theta_{n}^{\gamma} 2^{-k \gamma}\left(c_{7} 2^{\delta k} \log n\right)^{2 H(1+\gamma)} \\
& \leq c_{9} 2^{-n(1-H)} \sum_{k=1}^{\infty}\left(\log \log 2^{n}\right)^{-\gamma 2 H} 2^{-k \gamma}\left(c_{7} 2^{\delta k} \log n\right)^{2 H(1+\gamma)} \\
& \leq c_{10} 2^{-n(1-H)}\left(\log \log 2^{n}\right)^{2 H} \sum_{k=1}^{\infty} 2^{(\delta 2 H(1+\gamma)-\gamma) k} \\
& \leq c_{11} g\left(2^{-n}\right),
\end{aligned}
$$

where the last inequality follows from $\delta 2 H(1+\gamma)<\gamma$. Combining this with (4.1) then yields

$$
\sup _{|x| \leq 2 c_{1} 2^{-n H} \log n} L\left(x+Z_{s}, C_{n}\right) \leq c_{12} g\left(2^{-n}\right)
$$

or in other words,

$$
\sup _{\left|x-Z_{s}\right| \leq 2 c_{1} 2^{-n H} \log n} L\left(x, C_{n}\right) \leq c_{12} g\left(2^{-n}\right) .
$$

Claim (1.6) now follows from Step 1 and the fact $L^{*}\left(C_{n}\right)=\sup \left\{L\left(x, C_{n}\right): x \in\right.$ $\left.\overline{Z\left(C_{n}\right)}\right\}$.

Step 5: It remains to prove (1.7). However, for this the arguments are similar to above and to the Gaussian case (for detailed proof in the case of the fractional Brownian motion, we refer to [21]). Thus we present only the key arguments here. 
We choose $\tilde{\theta}_{n}=2^{-n H}\left(\log 2^{n}\right)^{-2 H}$ and

$$
\tilde{G}_{n}=\left\{x \in \mathbb{R}:|x| \leq n, x=\tilde{\theta}_{n} p, \text { for some } p \in \mathbb{Z}\right\} .
$$

If $\mathcal{D}_{n}$ is the dyadic partition of the interval $I$, then the arguments of Step 2 together with (3.8) gives that, for $n \geq n_{1}(\omega)$ and a suitable constant $c_{13}$, we have

$$
L(x, B) \leq c_{13} 2^{-n(1-H)}\left(\log 2^{n}\right)^{2 H}
$$

for all $B \in \mathcal{D}_{n}$ and $x \in \tilde{G}_{n}$. Similarly, in Step 3 and Step 4 we replace $F(n, k, x)$ with $\tilde{F}(n, k, x)$ where $G_{n}$ and $\theta_{n}$ are replaced with $\tilde{G}_{n}$ and $\tilde{\theta}_{n}$, and instead of $B_{n}$ we consider the event

$$
\begin{aligned}
& \tilde{B}_{n}=\left\{\left|L\left(y_{1}, B\right)-L\left(y_{2}, B\right)\right| \geq 2^{-n(1-H-\gamma H)}\left|y_{1}-y_{2}\right|^{\gamma}\left(c_{14} k \log 2^{n}\right)^{2 H(1+\gamma)}:\right. \\
& \left.\quad \text { for some } B \in \mathcal{D}_{n}, y_{1}, y_{2} \in \tilde{F}(n, k, x) \text { linked }\right\}
\end{aligned}
$$

for suitably chosen $\gamma$ and constant $c_{14}$. As in Step 3, then (3.9) gives that $\tilde{B}_{n}$ occurs only finitely many times. In the complement $\tilde{B}_{n}^{c}$ we can apply (4.2) and proceed as in Step 4 to conclude that for all $B \in \mathcal{D}_{n}$

$$
L(x, B) \leq c_{15} 2^{-n(1-H)}\left(\log 2^{n}\right)^{2 H} .
$$

This completes Step 5 and thus the whole proof.

Proofs of corollaries 1.5 to 1.7 follows essentially from Theorem 1.4 and the arguments presented in 2]. Thus we simply state the key arguments and leave the details to the reader.

Proof of Corollary 1.5. This follows directly from Theorem 1.4 and the fact that

$$
L(x,[t-r, t+r]) \leq L^{*}([t-r, t+r]) .
$$

Proof of Corollary [1.6. As the proof of [21, Theorem 4.1], the claim follows from Corollary 1.5 and the upper density theorem of [18]. We omit the details.

Proof of Corollary 1.7. This claim follows again from Theorem 1.4 by applying exactly the same arguments as in the proof of Theorem 4.5 of [2, Theorem 4.5]. We omit the details.

\section{Technical results}

\subsection{Spectral estimates.}

Proof of Lemma 2.1. Note that by (1.1) and (1.2),

$$
\sum_{j=1}^{n} \xi_{j} Z_{t_{j}}=\int_{\mathbb{R}^{2}} H_{t}(x, y) Z_{G}(d x) Z_{G}(d y),
$$


where the integral is taken over $x \neq \pm y, t=\left(t_{1}, \ldots, t_{n}\right) \in \mathbb{R}_{+}^{n}$ and

$$
H_{t}(x, y)=\sum_{j=1}^{n} \xi_{j} \frac{e^{i t_{j}(x+y)}-1}{i(x+y)}
$$

is a Hilbert-Schmidt kernel. Then the operator $A_{t, \xi}$ satisfies

$$
\left(A_{t, \xi} f\right)(x)=\int_{\mathbb{R}} H_{t}(x,-y) f(y) G(d y)=\int_{\mathbb{R}} \sum_{j=1}^{n} \xi_{j} \frac{e^{i t_{j}(x-y)}-1}{i(x-y)} f(y)|y|^{-H} d y .
$$

Similarly to (1.3), for all $\xi \in \mathbb{R}^{n}$,

$$
\sum_{j=1}^{n} \xi_{j} Z_{t_{j}} \stackrel{d}{=} \sum_{k=1}^{\infty} \lambda_{k}\left(X_{k}^{2}-1\right)
$$

where $\left(X_{k}\right)_{k \geq 1}$ is a sequence of independent Gaussian random variables and $\left(\lambda_{k}\right)_{k \geq 1}$ are the eigenvalues of the operator $A_{t, \xi}$. Then, the characteristic function (evaluated at 1) of $\sum_{j=1}^{n} \xi_{j} Z_{t_{j}}$ is

$$
\mathbb{E} \exp \left(i \sum_{j=1}^{n} \xi_{j} Z_{t_{j}}\right)=\prod_{k \geq 1} \frac{e^{-i \lambda_{k}}}{\sqrt{1-2 i \lambda_{k}}}
$$

where we have used the expression for the characteristic function of a $\chi^{2}$ distribution and independence. Note, that the product converges since $\sum \lambda_{k}^{2}<\infty$. Furthermore,

$$
\left|\mathbb{E} \exp \left(i \sum_{j=1}^{n} \xi_{j} Z_{t_{j}}\right)\right|=\prod_{k \geq 1} \frac{1}{\left(1+4 \lambda_{k}^{2}\right)^{1 / 4}},
$$

as desired.

Before we present the proof of Lemma 2.2 we will establish some properties of the convolution $K_{\alpha}$ defined via $\widehat{K_{\alpha} f}(\xi)=|\xi|^{-\alpha} \widehat{f}(\xi)$ for $\alpha \in[0,1 / 2)$. An alternative representation that is useful for the proof of Lemma 2.2 is

$$
K_{\alpha} f(x)=\int_{R} h(x-y) f(y) d y=\int_{R} k(x, y) f(y) d y,
$$

where $h(x-y)=k(x, y)=d_{\alpha}|x-y|^{\alpha-1}$ for some constant $d_{\alpha}$ (see e.g. [11, Theorem 2.4.6]).

Moreover, $K_{\alpha}$ can be extended to a bounded operator from $L^{2}(J)$ to $L^{2}(\mathbb{R})$ where $J \subset \mathbb{R}$ is compact. Let first $f \in C_{c}^{\infty}(\mathbb{R})$ and recall that the smooth compactly supported functions are dense in $L^{2}(J)$. Since $f$ is a Schwartz function, $\widehat{f}$ is bounded and decays at any polynomial rate. Then $\int_{\mathbb{R}}\left|\widehat{K_{\alpha} f}(\xi)\right|^{2} d \xi<\infty$, and by Plancherel's theorem $\int_{\mathbb{R}}\left|K_{\alpha} f(x)\right|^{2} d x<\infty$. 
We are left to show that when $f \in L^{2}(J), \int_{\mathbb{R}}\left|K_{\alpha} f(x)\right|^{2} d x<C(J) \| f||_{L^{2}(J)}^{2}$, for some constant $C(J)>0$ depending only on $J$. By Plancherel's theorem

$$
\begin{aligned}
\left\|K_{\alpha} f\right\|_{L^{2}(\mathbb{R})}^{2} & =\frac{1}{2 \pi} \int_{|\xi| \leq 1}|\xi|^{-2 \alpha}|\widehat{f}(\xi)|^{2} d \xi+\frac{1}{2 \pi} \int_{|\xi|>1}|\xi|^{-2 \alpha}|\widehat{f}(\xi)|^{2} d \xi \\
& \leq \int_{|\xi| \leq 1}|\xi|^{-2 \alpha}|\widehat{f}(\xi)|^{2} d \xi+\|f\|_{L^{2}(J)}^{2},
\end{aligned}
$$

To bound the first integral, note that by definition, for every $\xi \in \mathbb{R}$,

$$
|\widehat{f}(\xi)|=\left|\int_{J} e^{-i \xi x} f(x) d x\right| \leq \int_{J}|f(x)| d x \leq|J|^{1 / 2}\left(\int_{J}|f(x)|^{2}\right)^{1 / 2} d x
$$

where we have applied the Cauchy-Schwarz inequality, and $|J|$ is the Lebesgue measure of the compact set $J$. Therefore, combining (5.2) and (5.3), yields

$$
\left\|K_{\alpha} f\right\|_{L^{2}(\mathbb{R})}^{2} \leq\|f\|_{L^{2}(J)}^{2}\left(|J| \int_{-1}^{1}|\xi|^{-2 \alpha} d \xi+1\right)=C(J)\|f\|_{L^{2}(J)}^{2}<\infty,
$$

where $C(J)>0$ is some constant depending only on $J$. This establishes that $K_{\alpha}$ extends to a bounded operator from $L^{2}(J)$ to $L^{2}(\mathbb{R})$.

If $g$ is a bounded, compactly supported function and $M_{g}$ is the multiplication operator given by $M_{g} f(x)=g(x) f(x)$, then $M_{g}: L^{2}(\mathbb{R}) \rightarrow L^{2}(\mathbb{R})$ is obviously bounded and its (Hilbert space adjoint) equals $M_{\bar{g}}$. Our previous observation on $K_{\alpha}$ then implies that $K_{\alpha} M_{g}$ extends to a bounded operator on $L^{2}(\mathbb{R})$. Let us check that the adjoint of $K_{\alpha} M_{g}$ equals $M_{\bar{g}} K_{\alpha}$ (we need to be cautious since $K_{\alpha}$ is not bounded on the whole of $\left.L^{2}(\mathbb{R})\right)$ : for any $f, h \in C_{0}^{\infty}(\mathbb{R})$ we obtain

$$
\begin{aligned}
& \int_{\mathbb{R}} K_{\alpha} M_{g} f(x) \overline{h(x)} d x=\frac{1}{2 \pi} \int_{\mathbb{R}}\left(\widehat{K_{\alpha} M_{g}} f\right)(\xi) \overline{\widehat{h}(\xi)} d \xi=\frac{1}{2 \pi} \int_{\mathbb{R}} \widehat{g} * \widehat{f}(\xi) \overline{|\xi|-\alpha \widehat{h}(\xi)} d \xi \\
& =\frac{1}{2 \pi} \int_{\mathbb{R}} \int_{\mathbb{R}} \widehat{g}(\xi-t) \widehat{f}(t) d t \overline{\widehat{K_{\alpha} h}(\xi)} d \xi=\int_{\mathbb{R}} g(x) f(x) \overline{K_{\alpha} h(x)} d x \\
& =\int_{\mathbb{R}} f(x) \overline{M_{\bar{g}} K_{\alpha} h(x)} d x \text {. }
\end{aligned}
$$

Therefore, $M_{\bar{g}} K_{\alpha}$ extends to a bounded operator from $L^{2}(\mathbb{R})$ to $L^{2}(\mathbb{R})$, and by extension, so does $K_{\alpha} M_{g} K_{\alpha}$, since $K_{\alpha} M_{g} K_{\alpha}=K_{\alpha} M_{g}\left(K_{\alpha} M_{\chi_{J}}\right)^{*}$, where $J \subset \mathbb{R}$ is a compact interval containing the support of $g$.

Proof of Lemma 2.2. Let $T: L^{2}\left(|y|^{-H} d y\right) \rightarrow L^{2}(\mathbb{R})$ be given by $(T f)(x)=|x|^{-H / 2} f(x)$. Note that $T$ is an isometric isomorphism. Hence, the operator $A_{t, \xi}$ is isometrically isomorphic to $V_{t, \xi}:=T A_{t, \xi} T^{-1}: L^{2}(\mathbb{R}) \rightarrow L^{2}(\mathbb{R})$, that satisfies

$$
V_{t, \xi} f(x)=|x|^{-H / 2} \int_{\mathbb{R}} \sum_{j=1}^{n} \xi_{j} \frac{e^{i t_{j}(x-y)}-1}{i(x-y)} f(y)|y|^{-H / 2} d y .
$$


Next, recall that the Fourier transform $\mathcal{F}$ provides an isometric isomorphism $L^{2}(\mathbb{R}) \rightarrow$ $L^{2}(\mathbb{R})$, up to a constant, and transforms multiplication to convolution. Since

$$
(\mathcal{F} g)(x)=\left(\sum_{j=1}^{n} \xi_{j} \mathcal{F} \chi_{\left[0, t_{j}\right]}\right)(x)=\sum_{j=1}^{n} \xi_{j} \frac{e^{-i t_{j} x}-1}{-i x}
$$

it follows that

$$
\begin{aligned}
\mathcal{F}^{3}\left(K_{H / 2} M_{g} K_{H / 2} f\right)(x) & =2 \pi \mathcal{F}\left(K_{H / 2} M_{g} K_{H / 2} f\right)(-x)=|x|^{-H / 2}\left((\mathcal{F} g) * \widehat{K_{H / 2} f}\right)(-x) \\
& =2 \pi|x|^{-H / 2} \int_{\mathbb{R}} \sum_{j=1}^{n} \xi_{j} \frac{e^{-i t_{j}(-x-y)}-1}{-i(-x-y)} \widehat{f}(y)|y|^{-H / 2} d y, \\
& =2 \pi|x|^{-H / 2} \int_{\mathbb{R}} \sum_{j=1}^{n} \xi_{j} \frac{e^{i t_{j}(x-y)}-1}{i(x-y)} \widehat{f}(-y)|y|^{-H / 2} d y, \\
& =|x|^{-H / 2} \int_{\mathbb{R}} \sum_{j=1}^{n} \xi_{j} \frac{e^{i t_{j}(x-y)}-1}{i(x-y)}\left(\mathcal{F}^{3} f\right)(y)|y|^{-H / 2} d y, \\
& =\left(V_{t, \xi} \mathcal{F}^{3} f\right)(x) .
\end{aligned}
$$

where $*$ denotes convolution, and we have used that $\mathcal{F}^{3} f(x)=2 \pi \widehat{f}(-x)$. Therefore, there is a constant $c(H)$ depending only on $H$, such that $A_{t, \xi}$ and $B(t, \xi):=$ $c(H) K_{H / 2} M_{g} K_{H / 2}$ are unitarily equivalent. In particular, since $A_{t, \xi}$ is self-adjoint, so is $B(t, \xi)$, although this can be easily seen also from the definition of $B$. Moreover, the two operators have the same eigenvalues, and, more importantly, their singular value sequences coincide.

In order to establish (2.2) we need two technical results. First, a key Lemma 5.1 compares the singular values of $B_{t, \xi}$ to $B_{t_{1}, \xi_{1}}=c(H) \xi_{1} K_{H / 2} \chi_{\left[0, t_{1}\right]} K_{H / 2}$. Then, Lemma 5.4, establishes asymptotics for the singular values of a related operator.

Lemma 5.1. Let $\alpha \in(0,1 / 2)$ and $I \subset \mathbb{R}$ be an interval. Then, there is a bounded operator $U_{\alpha, I}$ on $L^{2}(\mathbb{R})$, whose norm is bounded by a finite constant that depends only on $\alpha$, so that for any compactly supported $f \in L^{2}(\mathbb{R})$ :

$$
K_{\alpha}\left(M_{\chi_{I}} f\right)=U_{\alpha, I} K_{\alpha} f .
$$

Proof. We have shown that if $f \in L^{2}(\mathbb{R})$ is compactly supported, then $K_{\alpha} f \in L^{2}(\mathbb{R})$. Therefore, we need to show that for all $h \in L^{2}(\mathbb{R})$, the operator $U_{\alpha, I}$, defined a priori only on $C_{0}^{\infty}(\mathbb{R})$

$$
U_{\alpha, I} h:=K_{\alpha} M_{\chi_{I}} K_{-\alpha} h,
$$

extends to a well defined bounded operator from $L^{2}(\mathbb{R})$ to $L^{2}(\mathbb{R})$ (note that the factor $K_{-\alpha}$ alone does not have this property, but $K_{-\alpha} f \in L^{2}(\mathbb{R})$ for $f \in C_{0}^{\infty}(\mathbb{R})$ ).

Again we use that $C_{c}^{\infty}(\mathbb{R})$ is dense in $L^{2}(\mathbb{R})$. Let $h \in C_{c}^{\infty}(\mathbb{R})$. Then, as before, by the Paley-Wiener theorem and the Plancherel's theorem, $U_{\alpha, I}$ is a well-defined 
operator from $C_{c}^{\infty}(\mathbb{R})$ to $L^{2}(\mathbb{R})$, and $(\underline{5.5})$ holds. To extend it to all of $L^{2}(\mathbb{R})$, we show the following a priori norm bound

$$
\int_{\mathbb{R}}\left|K_{\alpha} M_{\chi_{I}} K_{-\alpha} h\right|^{2} \leq c(\alpha) \int_{\mathbb{R}}|h|^{2}
$$

for $h=K_{\alpha} f$, where $f \in C_{0}^{\infty}(\mathbb{R})$ - such functions are clearly dense in $L^{2}(\mathbb{R})$. Note, that $f=K_{-\alpha} h$. From Parseval identity we get

$$
\int_{\mathbb{R}}\left|K_{\alpha} M_{\chi_{I}} K_{-\alpha} f\right|^{2}=\int_{\mathbb{R}}\left|K_{\alpha} M_{\chi_{I}} f\right|^{2}=(2 \pi)^{-1} \int_{\mathbb{R}}|\xi|^{-2 \alpha}\left|\widehat{M_{\chi_{I}} f}(\xi)\right|^{2} d \xi,
$$

and, by the convolution theorem,

$$
\widehat{M_{\chi_{I}} f}=(2 \pi)^{-1} \widehat{\chi_{I}} * \widehat{f},
$$

so everything is well-defined as $\widehat{f}$ decays at any polynomial rate. The right-hand side of (5.6) can be written as

$$
\int_{\mathbb{R}}|h|^{2}=\int_{\mathbb{R}}\left|K_{\alpha} f\right|^{2}=\int_{\mathbb{R}}|\xi|^{-2 \alpha}|\widehat{f}(\xi)|^{2} d \xi
$$

To establish (5.6), we need to show

$$
\int_{\mathbb{R}}|\xi|^{-2 \alpha}\left|\widehat{M_{\chi_{I}} f}(\xi)\right|^{2} d \xi \leq c(\alpha) \int_{\mathbb{R}}|\xi|^{-2 \alpha}|\widehat{f}(\xi)|^{2} d \xi .
$$

We recall some properties of the Hilbert transform (see [11], [9]), defined (at least) for test functions as the singular value integral

$$
\mathcal{H} g(x):=\lim _{\varepsilon \rightarrow 0} \frac{1}{\pi} \int_{|x-y| \geq \varepsilon} \frac{g(y)}{x-y} d y,
$$

where $g \in L^{2}(\mathbb{R})$. First, the Hilbert transform has a bounded extension to $L^{2}(\mathbb{R})$. The boundedness is most easily seen by the fact that it is a multiplier operator with bounded symbol:

$$
\mathcal{F}(\mathcal{H} g)(\xi)=(-i \operatorname{sgn}(\xi)) \widehat{g}(\xi)
$$

Moreover (see [9, Section 3.5]),

$$
\mathcal{F}\left(\frac{i}{2}\left(N_{a} \mathcal{H} N_{-a}-N_{b} \mathcal{H} N_{-b}\right) g\right)(\xi)=\chi_{(a, b)}(\xi) \widehat{g}(\xi),
$$

where $N_{a}$ is the isometric multiplication operator given by $N_{a}=M_{e^{i a x}}$. Applying $\mathcal{F}^{-1}$ to the above (and recalling that $\mathcal{F}^{-1} f(\xi)=\frac{1}{2 \pi} \widehat{f}(-\xi)$ ) yields

$$
\begin{aligned}
\left(\frac{i}{2}\left(N_{a} \mathcal{H} N_{-a}-N_{b} \mathcal{H} N_{-b}\right) g\right)(\xi) & =\frac{1}{2 \pi} \int_{\mathbb{R}} \widehat{\chi_{(a, b)}}(-y) g(x-y) d y \\
& =\frac{1}{2 \pi} \widehat{\chi_{(-b, a)}} * g(\xi) .
\end{aligned}
$$


In particular, if $I=(-b,-a)$, we obtain up to an absolute constant,

$$
\left|\left(\frac{i}{2}\left(N_{a} \mathcal{H} N_{-a}-N_{b} \mathcal{H} N_{-b}\right) \widehat{f}\right)(\xi)\right|^{2}=c\left|\widehat{M_{\chi_{I}} f}(\xi)\right|^{2} .
$$

Then, since $N_{a}$ is isometric in the weighted space, in order to establish (5.7) it suffices to show that

$$
\int_{\mathbb{R}}|\mathcal{H} \widehat{f}(\xi)|^{2}|\xi|^{-2 \alpha} d \xi \leq c(\alpha) \int_{\mathbb{R}}|\widehat{f}(\xi)|^{2}|\xi|^{-2 \alpha} d \xi
$$

At this point we recall a more general result due to Hunt et al [14].

Proposition 5.2. If $1<p<\infty$ and $W(x)$ is nonnegative, the following are equivalent.

(1) There is a constant $C$ independent of $I$, such that for every interval $I$,

$$
\left[\frac{1}{|I|} \int_{I} W(x) d x\right]\left[\frac{1}{|I|} \int_{I} W(x)^{-1 /(p-1)} d x\right]^{p-1} \leq C .
$$

(2) There is a constant $C$, independent of $f$, such that

$$
\int_{-\infty}^{\infty}|\mathcal{H} f(x)|^{p} W(x) d x \leq C \int_{-\infty}^{\infty}|f(x)|^{p} W(x) d x
$$

Nonnegative functions $W(x)$ that satisfy (5.9) are called $A_{p}$ weights. We can apply (5.10) in order to establish (5.8) provided that $|x|^{-2 \alpha}$ is an $A_{2}$ weight. This fact is established for $\alpha \in(0,1 / 2)$ in, e.g., [12, Example 9.1.7.]

Remark 5.3. The previous lemma is crucial in our estimation of the singular values, since it verifies that the singular values will dominate those of any localization of the multiplier $g$. This is not at all obvious, since $g$ typically changes sign in our situation, and a potential cancellation phenomenon could prevent the needed estimate.

We now state the second technical lemma.

Lemma 5.4. Let $\alpha \in(0,1 / 2)$ and $J \subset \mathbb{R}$ be a finite interval. Then the operator $\mathcal{M}_{\alpha, J}:=M_{\chi_{J}} K_{\alpha} M_{\chi_{J}}$ is bounded, self-adjoint, and positive, and its singular value sequence is of the form $\left(|J|^{\alpha} \widetilde{\mu}_{k}\right)_{k \geq 1}$, where $\left(\widetilde{\mu}_{k}\right)_{k \geq 1}$ is the singular value sequence of $\mathcal{M}_{\alpha,[0,1]}$. Moreover, $\widetilde{\mu}_{k}>0$ for all $k \geq 1$, and

$$
\widetilde{\mu}_{k} \sim c k^{-\alpha} \quad \text { as } \quad k \rightarrow \infty .
$$

Proof. Recall that $K_{\alpha} M_{\chi_{J}}$ is bounded since $J$ is a finite interval, and note that $M_{\chi_{J}}$ is an orthogonal projection and has norm 1, especially $M_{\chi_{J}}^{2}=M_{\chi_{J}}$ and $M_{\chi_{J}}^{*}=M_{\overline{\chi_{J}}}=$ $M_{\chi_{J}}$. Thus $\mathcal{M}_{\alpha, J}$ is bounded. Finally, by Plancherel's theorem, for any $f, g \in L^{2}(\mathbb{R})$

$$
\int_{\mathbb{R}}\left(M_{\chi_{J}} K_{\alpha} M_{\chi_{J}} f\right)(x) \overline{g(x)} d x=\int_{\mathbb{R}}\left(K_{\alpha} M_{\chi_{J}} f\right)(x) \overline{M_{\chi_{J}} g(x)} d x
$$




$$
=\int_{\mathbb{R}}|\xi|^{-\alpha} \widehat{\overline{M_{\chi_{J}} f}}(\xi) \overline{\widehat{M_{\chi_{J}}} g}(\xi) d \xi .
$$

This shows that $\mathcal{M}_{\alpha, J}$ is self-adjoint and positive (take $g=f$ above).

To establish the relation of the singular values of $\mathcal{M}_{\alpha, J}$ to the ones of $\mathcal{M}_{\alpha, J}$ we will use the fact that the spectrum of two unitarily equivalent operators is identical. First, assume $J=[b, c]$ and let $\tau_{b} f(x):=f(x-b)$ be the translation by $b$. The operator $\tau_{b}$ is unitary with an adjoint given by $\tau_{-b} f(x)=f(x+b)$. Then, note that

$$
\begin{aligned}
\tau_{-b} \mathcal{M}_{\alpha,[b, c]} \tau_{b} f(x) & =\chi_{[b, c]}(x+b) \int_{\mathbb{R}} h(x+b-y) \chi_{[b, c]}(y) f(y-b) d y \\
& =\chi_{[0, c-b]}(x) \int_{\mathbb{R}} h\left(x-y^{\prime}\right) \chi_{[0, c-b]}\left(y^{\prime}\right) f\left(y^{\prime}\right) d y^{\prime} \\
& =\mathcal{M}_{\alpha,[0, c-b]}(x) .
\end{aligned}
$$

Therefore, the singular values of $\mathcal{M}_{\alpha,[b, c]}$ are the same as the ones of $\mathcal{M}_{\alpha,[0, c-b]}$. Next, set $J=[0, b]$ and consider the rescaling unitary operator $\mu_{b} f(x):=\sqrt{b} f(b x)$ with an inverse given by $\mu_{b}^{-1} f(x)=f(x / b) / \sqrt{b}$. Now,

$$
\begin{aligned}
\mu_{b} \mathcal{M}_{\alpha,[0, b]} \mu_{b}^{-1} f(x) & =\sqrt{b} \chi_{[0, b]}(b x) \int_{\mathbb{R}} h(b x-y) \chi_{[0, b]}(y) f(y / b) / \sqrt{b} d y \\
& =\chi_{[0,1]}(x) \int_{\mathbb{R}} h\left(b\left(x-y^{\prime}\right)\right) \chi_{[0,1]}\left(y^{\prime}\right) f\left(y^{\prime}\right) b d y^{\prime} \\
& =b^{\alpha} \mathcal{M}_{\alpha,[0,1]}(x),
\end{aligned}
$$

where we have used that $h(b z)=b^{\alpha-1} h(z)$. Thus, the correspondence between the singular values of $\mathcal{M}_{\alpha, J}$ and $\mathcal{M}_{\alpha,[0,1]}$ is established.

Next, for the proof of the decay of the singular values of $\mathcal{M}_{\alpha,[0,1]}$, we need to show that $\mathcal{M}_{\alpha, J}$ is a compact operator. Recall (5.1):

$$
K_{\alpha} f(x)=\int_{\mathbb{R}} h(x-y) f(y) d y=\int_{\mathbb{R}} k(x, y) f(y) d y .
$$

Integral operators are compact if $\int_{\mathbb{R}^{2}}|k(x, y)|^{2} d x d y<\infty$. However, for the operator $\mathcal{M}_{\alpha, J}$ one only needs to show that $\int_{J^{2}}|k(x, y)|^{2} d x d y<\infty$ which is indeed the case for $k(x, y)=d_{\alpha}|x-y|^{\alpha-1}$ with $\alpha>1 / 2$.

To finish the proof, recall a result by Dostanic [7, Theorem 1] where the decay of the singular values is established for $\mathcal{M}_{\alpha,[-1,1]} / d_{\alpha}$.

Proposition 5.5. Let $A: L^{2}(-1,1) \rightarrow L^{2}(-1,1)$ be the self-adjoint operator defined by

$$
A f(x)=\int_{-1}^{1}|x-y|^{\alpha-1} f(y) d y, \quad 0<\alpha<1 .
$$

Then, the eigenvalues of $A$ are simple and satisfy $\lambda_{n}(A) \sim c n^{-\alpha}$ with a constant $c=c($ alpha $)>0$. 
Next, we finish the proof of (2.2). We have $t_{0}=0<t_{1}<\cdots<t_{n} \leq 1$. Set $I_{j}=\left[t_{j-1}, t_{j}\right]$ for $1 \leq j \leq n$. Then $g(x)=\sum_{j=1}^{n}\left(\xi_{j}-\xi_{j-1}\right) \chi_{I_{j}}$. Fix $j \in[1, n]$ and let $\left(\left\|U_{\alpha, I_{j}}\right\|\right)^{-1} \geq C(\alpha)>0$ with $U_{\alpha, I_{j}}$ as in Lemma 5.1. Recall that if $A$ and $B$ are bounded operators on $L^{2}(\mathbb{R})$, then by the Minimax principle [4, Corollary III.1.2],

$$
\mu_{n}(A B)=\max _{\substack{M \subset L^{2}(\mathbb{R}) \\ \operatorname{dim} M=n}} \min _{\substack{x \in M \\\|x\|=1}}\|A B x\|
$$

and then $\mu_{n}(A B) \leq\|A\| \mu_{n}(B)$ and $\mu_{n}(A B) \leq\|B\| \mu_{n}(A)$. Therefore,

$$
\mu_{n}\left(K_{\alpha} M_{g} K_{\alpha}\right) \geq\left(\left\|U_{\alpha, I_{j}}\right\|\right)^{-1} \mu_{n}\left(U_{\alpha, I_{j}} K_{\alpha} M_{g} K_{\alpha}\right)=C(\alpha) \mu_{n}\left(K_{\alpha} M_{\chi_{I_{j}}} M_{g} K_{\alpha}\right) .
$$

Moreover,

$$
\mu_{n}\left(K_{\alpha} M_{\chi_{I_{j}}} M_{g} K_{\alpha}\right)=\mu_{n}\left(K_{\alpha}\left(\left(\xi_{j}-\xi_{j-1}\right) M_{\chi_{I_{j}}}\right) K_{\alpha}\right)=\left|\xi_{j}-\xi_{j-1}\right| \mu_{n}\left(K_{\alpha} M_{\chi_{I_{j}}} K_{\alpha}\right) .
$$

Then, since $\left\|M_{\chi_{I_{j}}}\right\|=1$ and $M_{\chi_{I_{j}}}^{2}=M_{\chi_{I_{j}}}$ we have

$$
\mu_{n}\left(K_{\alpha} M_{\chi_{I_{j}}} K_{\alpha}\right) \geq \mu_{n}\left(M_{\chi_{I_{j}}} K_{\alpha} M_{\chi_{I_{j}}} K_{\alpha} M_{\chi_{I_{j}}}\right)=\mu_{n}\left(\left(M_{\chi_{I_{j}}} K_{\alpha} M_{\chi_{I_{j}}}\right)^{2}\right) .
$$

Next, by Lemma 5.4

$$
\mu_{n}\left(\left(M_{\chi_{I_{j}}} K_{\alpha} M_{\chi_{I_{j}}}\right)^{2}\right)=\mu_{n}\left(M_{\chi_{I_{j}}} K_{\alpha} M_{\chi_{I_{j}}}\right)^{2}=\left|t_{j}-t_{j-1}\right|^{2 \alpha}\left(\widetilde{\mu}_{n}\right)^{2},
$$

where we have used that if $A$ is self-adjoint, $\mu_{n}\left(A^{2}\right)=\mu_{n}(A)^{2}$. Therefore,

$$
\mu_{n}\left(K_{\alpha} M_{g} K_{\alpha}\right) \geq C(\alpha) \max _{1 \leq j \leq n}\left|\xi_{j}-\xi_{j-1}\right|\left|t_{j}-t_{j-1}\right|^{2 \alpha}\left(\widetilde{\mu}_{n}\right)^{2},
$$

where $\widetilde{\mu}_{n} \sim n^{-\alpha}$. Finally, (2.2) follows with $\alpha=H / 2$.

\subsection{Integral estimates.}

Proof of Lemma 2.3. Note, that for any $N>0$,

$$
1+\sum_{k=1}^{N} 4 s^{2} \widetilde{\mu}_{k}^{4} \leq \prod_{k=1}^{N}\left(1+4 s^{2} \widetilde{\mu}_{k}^{4}\right) \leq \exp \left(\sum_{k=1}^{N} 4 s^{2} \widetilde{\mu}_{k}^{4}\right) .
$$

By Lemma 5.4, $\widetilde{\mu}_{k}^{4} \approx k^{-2 H}$ and $\sum_{k=1}^{\infty} 4 s^{2} \widetilde{\mu}_{k}^{4}<\infty$ since $H \in(1 / 2,1)$. Therefore, $G(s)$ converges and also $G(s)>0$. Next, let $a>0$ be such that $4 \widetilde{\mu}_{k}^{4} \geq a k^{-2 H}$ for all $k \geq 1$ and set $z=a s^{2}$. Then, the elementary inequality $\log (1+x) \geq x / 2$ for $x \in[0,1)$ yields for $z \geq 1$ that

$$
\begin{aligned}
-8 \log G(s) & =2 \sum_{k=1}^{\infty} \log \left(1+4 s^{2} \widetilde{\mu}_{k}^{4}\right) \geq 2 \sum_{k=1}^{\infty} \log \left(1+z k^{-2 H}\right) \geq z \sum_{k \geq z^{1 / 2 H}} k^{-2 H} \\
& \geq z \int_{2 z^{1 / 2 H}} x^{-2 H}=2^{1-2 H}(2 H-1)^{-1} z^{1 / 2 H} \geq c_{0} z^{1 / 2 H},
\end{aligned}
$$


where $c_{0}>0$, and in general $-8 \log G(s) \geq c_{0}\left(z^{1 / 2 H}-1\right)$. Thus, $G(s) \leq c_{2} \exp \left(-c_{1} s^{1 / H}\right)$, where $c_{1}, c_{2}>0$, and we obtain

$$
\begin{aligned}
\int_{0}^{\infty} s^{\beta-1} G(s) d s & \leq \int_{0}^{\infty} c_{2} \exp \left(-c_{1} s^{1 / H}\right) s^{\beta-1} d s=c_{2} H \int_{0}^{\infty} \exp \left(-c_{1} x\right) x^{\beta H-1} d x \\
& =c_{2} H c_{1}^{-\beta H} \Gamma(\beta H) \leq c_{3}^{\beta H} \Gamma(\beta H) .
\end{aligned}
$$

as desired.

Proof of Lemma 2.4. Our goal is to estimate the integral

$$
I_{0}:=\int_{S^{n-1}} \int_{\substack{t_{1}+t_{2}+\ldots+t_{n} \leq 1 \\ t_{1}, \ldots, t_{n} \geq 0}} \prod_{j=1}^{n}\left|y_{j}\right|^{\gamma_{j}}(f(t, y))^{-n\left(1+\gamma_{\mathrm{av}}\right)} d t \mathcal{H}^{n-1}(d y),
$$

where $f: \mathbb{R}_{+}^{n} \times \mathbb{R}^{n} \rightarrow(0, \infty)$ is given by

$$
f(t, y)=t_{1}^{H}\left|y_{1}\right| \vee t_{2}^{H}\left|y_{2}\right| \vee \cdots \vee t_{n}^{H}\left|y_{n}\right|,
$$

and $\gamma_{\mathrm{av}}:=n^{-1} \sum_{j=1}^{n} \gamma_{n}$ for $\gamma_{1}, \ldots \gamma_{n} \geq 0$. First note that $f$ is $H$-homogeneous with respect to $t$ and 1 -homogeneous with respect to $y$ :

$$
f(t, y)=|t|^{H}|y| f\left(t^{0}, y^{0}\right),
$$

where $y^{0}:=y /|y|$ and $t^{0}:=t /\left|\sum_{j=1}^{n} t_{j}\right|$. Observe that we use the standard Euclidean norm for the $y$-variable and the $\ell^{1}$-norm for the $t$-variable. Next, introduce the related integral

$$
I_{1}:=\int_{S^{n-1}} \int_{\substack{t_{1}+t_{2}+\ldots+t_{n}=1 \\ t_{1}, \ldots, t_{n} \geq 0}} \prod_{j=1}^{n}\left|y_{j}\right|^{\gamma_{j}}(f(t, y))^{-n\left(1+\gamma_{\mathrm{av}}\right)} \mathcal{H}^{n-1}(d t) \mathcal{H}^{n-1}(d y)
$$

Using the $H$-homogeneity of $f$ with respect to $t$ and noting that the distance of the origin from the hyperplane $t_{1}+\ldots+t_{n}=1$ equals $n^{-1 / 2}$, we obtain the following relation between $I_{0}$ and $I_{1}$ :

$$
I_{0}=I_{1} \int_{0}^{n^{-1 / 2}}\left(u / n^{-1 / 2}\right)^{-n\left(1+\gamma_{\mathrm{av}}\right) H}\left(u / n^{-1 / 2}\right)^{n-1} d u,
$$

where the term $\left(u / n^{-1 / 2}\right)^{n-1}$ arises from the Jacobian and the rescaling. The above can be further simplified:

$$
I_{0}=I_{1} n^{-1 / 2} \int_{0}^{1} v^{n\left(1-H\left(1+\gamma_{\mathrm{av}}\right)\right)-1} d v=\left(1-H\left(1+\gamma_{\mathrm{av}}\right)\right)^{-1} n^{-3 / 2} I_{1},
$$

where one naturally needs to assume that $H\left(1+\gamma_{\text {av }}\right)<1$.

We proceed by showing the following more general result. 
Lemma 5.6. Assume that $f: \mathbb{R}_{+}^{n} \times \mathbb{R}^{n} \rightarrow(0, \infty)$ satisfies (5.11). Let $H \in(0,1)$ and $\gamma:=\left(\gamma_{1}, \ldots, \gamma_{n}\right) \in \mathbb{R}$ with $0 \leq \gamma_{j}<H^{-1}-1$ for every $j=1, \ldots, n$. Set $\gamma_{\mathrm{av}}:=n^{-1} \sum_{j=1}^{n} \gamma_{j}$. Then

$$
I_{0}=C(n, \gamma, H) \int_{\left(\mathbb{R}_{+}\right)^{n} \times \mathbb{R}^{n}} e^{-f(t, y)} e^{-\left(t_{1}+\ldots+t_{n}\right)} \prod_{j=1}^{n}\left|y_{j}\right|^{\gamma_{j}} d y d t
$$

where

$$
C(n, \gamma, H):=\frac{1}{n^{1 / 2} \Gamma\left(n\left(1+\gamma_{\mathrm{av}}\right)\right) \Gamma\left(n\left(1-H\left(1+\gamma_{\mathrm{av}}\right)+1\right)\right.} .
$$

Moreover, if $f$ is such that

$$
f(y, t)=g\left(t_{1}^{H}\left|y_{1}\right|, \ldots, t_{n}^{H}\left|y_{n}\right|\right),
$$

where $g$ is 1-homogeneous, then

$$
I_{0}=C(n, \gamma, H) \prod_{j=1}^{n} \Gamma\left(1-H\left(1+\gamma_{j}\right)\right) \int_{\mathbb{R}^{n}} e^{-g\left(\left|y_{1}\right|, \ldots,\left|y_{n}\right|\right)}\left|y_{1}\right|^{\gamma_{1}} \cdots\left|y_{n}\right|^{\gamma_{n}} d y
$$

Proof. We first compute $I_{1}$ by moving to radial variables in $y$ and $t$. Thus, let $r:=|y|, w:=y / r, s:=t_{1}+t_{2}+\ldots+t_{n}$ and $u:=t / s$ and note as a first step that

$$
\begin{aligned}
& \int_{\left(\mathbb{R}_{+}\right)^{n} \times \mathbb{R}^{n}} e^{-f(t, y)} e^{-\left(t_{1}+\ldots+t_{n}\right)}\left|y_{1}\right|^{\gamma_{1}} \cdots\left|y_{n}\right|^{\gamma_{n}} d y d t \\
& =\int_{|w|=1} \int_{\substack{u_{1}+u_{2}+\ldots+u_{n}=1 \\
u_{1}, \ldots, u_{n} \geq 0}} \int_{0}^{\infty} \int_{0}^{\infty} e^{-r s^{H} f(u, w)} e^{-s}\left|w_{1}\right|^{\gamma_{1}} \cdots\left|w_{n}\right|^{\gamma_{n}} r^{n \gamma_{\mathrm{av}}} \\
& s^{n-1} r^{n-1} d r d s \mathcal{H}^{n-1}(d u) \mathcal{H}^{n-1}(d w)
\end{aligned}
$$

We compute first the integral with respect to $r$ by making a change of variables $r^{\prime}:=r s^{H} f(u, t)$ and then with respect to $s$ to obtain

$$
\begin{aligned}
& \int_{0}^{\infty} \int_{0}^{\infty} e^{-r s^{H} f(u, w)} e^{-s} s^{n-1} d s r^{n\left(1+\gamma_{\mathrm{av}}\right)-1} d r \\
= & \int_{0}^{\infty} e^{-s}(f(u, w))^{-n\left(1+\gamma_{\mathrm{av}}\right)} s^{n\left(1-H\left(1+\gamma_{\mathrm{av}}\right)\right)-1} \int_{0}^{\infty} e^{-r^{\prime}}\left(r^{\prime}\right)^{n\left(1+\gamma_{\mathrm{av}}\right)-1} d r^{\prime} d s \\
= & \Gamma\left(n\left(1+\gamma_{\mathrm{av}}\right)\right)(f(u, w))^{-n\left(1+\gamma_{\mathrm{av}}\right)} \int_{0}^{\infty} e^{-s} s^{n\left(1-H\left(1+\gamma_{\mathrm{av}}\right)\right)-1} d s \\
= & \Gamma\left(n\left(1+\gamma_{\mathrm{av}}\right)\right) \Gamma\left(\left(1-H\left(1+\gamma_{\mathrm{av}}\right)\right) n\right)(f(u, w))^{-n\left(1+\gamma_{\mathrm{av}}\right)} .
\end{aligned}
$$

Next, recall that $I_{1}$ is given by

$$
I_{1}=\int_{|w|=1} \int_{\substack{u_{1}+u_{2}+\ldots+u_{n}=1 \\ u_{1}, \ldots, u_{n} \geq 0}}\left|w_{1}\right|^{\gamma_{1}} \cdots\left|w_{n}\right|^{\gamma_{n}}(f(u, w))^{-n\left(1+\gamma_{\mathrm{av}}\right)} \mathcal{H}^{n-1}(d u) \mathcal{H}^{n-1}(d w) .
$$


Therefore,

$$
\begin{aligned}
& \int_{\left(\mathbb{R}_{+}\right)^{n} \times \mathbb{R}^{n}} e^{-f(t, y)} e^{-\left(t_{1}+\ldots+t_{n}\right)}\left|y_{1}\right|^{\gamma_{1}} \cdots\left|y_{n}\right|^{\gamma_{n}} d y d t \\
= & \Gamma\left(n\left(1+\gamma_{\mathrm{av}}\right)\right) \Gamma\left(\left(1-H\left(1+\gamma_{\mathrm{av}}\right)\right) n\right) I_{1} \\
= & \Gamma\left(n\left(1+\gamma_{\mathrm{av}}\right)\right) \Gamma\left(\left(1-H\left(1+\gamma_{\mathrm{av}}\right)\right) n\right)\left(1-H\left(1+\gamma_{\mathrm{av}}\right)\right) n^{3 / 2} I_{0} \\
= & C(n, \gamma, H)^{-1} I_{0},
\end{aligned}
$$

where we have used the relation (5.12).

To establish (5.14) note that with the change of variables $y_{j}=u_{j} / t_{j}^{H}$,

$$
\begin{aligned}
& \int_{\left(\mathbb{R}_{+}\right)^{n} \times \mathbb{R}^{n}} e^{-g\left(t_{1}^{H}\left|y_{1}\right|, \ldots, t_{n}^{H}\left|y_{n}\right|\right)} e^{-\left(t_{1}+\ldots+t_{n}\right)}\left|y_{1}\right|^{\gamma_{1}} \cdots\left|y_{n}\right|^{\gamma_{n}} d y d t \\
= & \int_{\left(\mathbb{R}_{+}\right)^{n} \times \mathbb{R}^{n}} e^{-g\left(\left|u_{1}\right|, \ldots,\left|u_{n}\right|\right)} e^{-\left(t_{1}+\ldots+t_{n}\right)}\left|u_{1}\right|^{\gamma_{1}} \cdots\left|u_{n}\right|^{\gamma_{n}} \prod_{j=1}^{n} t_{j}^{-H\left(1+\gamma_{j}\right)} d u d t \\
= & \prod_{j=1}^{n} \Gamma\left(1-H\left(1+\gamma_{j}\right)\right) \int_{\mathbb{R}^{n}} e^{-g\left(\left|u_{1}\right|, \ldots,\left|u_{n}\right|\right)}\left|u_{1}\right|^{\gamma_{1}} \ldots\left|u_{n}\right|^{\gamma_{n}} \prod_{j=1}^{n} t_{j}^{-H\left(1+\gamma_{j}\right)} d u,
\end{aligned}
$$

and the conclusion follows after an application of (5.13).

Finally, we establish (2.6). By homogeneity,

$$
\int_{\partial([-u, u])} \prod_{j=1}^{n}\left|y_{j}\right|^{\gamma_{j}} \mathcal{H}^{n-1}(d y)=u^{n \gamma_{\mathrm{av}}} u^{n-1} \int_{\partial\left([-1,1]^{n}\right)} \prod_{j=1}^{n}\left|y_{j}\right|^{\gamma_{j}} \mathcal{H}^{n-1}(d y) .
$$

Next, by Fubini theorem,

$$
\int_{[-1,1]^{n}} \prod_{j=1}^{n}\left|y_{j}\right|^{\gamma_{j}} d y=\frac{1}{n\left(1+\gamma_{\mathrm{av}}\right)} \int_{\partial\left([-1,1]^{n}\right)} \prod_{j=1}^{n}\left|y_{j}\right|^{\gamma_{j}} \mathcal{H}^{n-1}(d y)=: \frac{A}{n\left(1+\gamma_{\mathrm{av}}\right)}
$$

where $A$ is the integral over the boundary. Moreover,

$$
\int_{[-1,1]^{n}} \prod_{j=1}^{n}\left|y_{j}\right|^{\gamma_{j}} d y=\prod_{j=1}^{n} \int_{[-1,1]}\left|y_{j}\right|^{\gamma_{j}} d y_{j}=\prod_{j=1}^{n} \frac{2}{1+\gamma_{j}} .
$$

Next, consider

$$
\begin{aligned}
& \int_{\mathbb{R}^{n}} e^{-\max _{1 \leq j \leq n}\left|y_{j}\right|} \prod_{j=1}^{n}\left|y_{j}\right|^{\gamma_{j}} d y \\
= & \int_{0}^{\infty} \int_{\partial([-u, u])} e^{-\max _{1 \leq j \leq n}\left|y_{j}\right|} \prod_{j=1}^{n}\left|y_{j}\right|^{\gamma_{j}} \mathcal{H}^{n-1}(d y) d u \\
= & \int_{0}^{\infty} e^{-u} \int_{\partial([-u, u])} \prod_{j=1}^{n}\left|y_{j}\right|^{\gamma_{j}} \mathcal{H}^{n-1}(d y) d u
\end{aligned}
$$




$$
\begin{aligned}
& =\int_{0}^{\infty} e^{-u} A u^{n-1} u^{n \gamma_{\mathrm{av}}} d u \\
& =n\left(1+\gamma_{\mathrm{av}}\right) \Gamma\left(n\left(1+\gamma_{\mathrm{av}}\right)\right) \prod_{j=1}^{n} \frac{2}{1+\gamma_{j}} .
\end{aligned}
$$

Finally, applying (5.15) in (5.14) with $g\left(\left|y_{1}\right|, \ldots,\left|y_{n}\right|\right)=\max _{1 \leq j \leq n}\left|y_{j}\right|$ yields

$$
\begin{aligned}
& \int_{S^{n-1}} \int_{\substack{t_{1}+t_{2}+\ldots+t_{n} \leq 1 \\
t_{1}, \ldots, t_{n} \geq 0}} \prod_{j=1}^{n}\left|y_{j}\right|^{\gamma_{j}}(f(t, y))^{-n\left(1+\gamma_{\mathrm{av}}\right)} d t \mathcal{H}^{n-1}(d y) \\
= & \frac{n^{1 / 2}\left(1+\gamma_{\mathrm{av}}\right)}{\Gamma\left(n\left(1-H\left(1+\gamma_{\mathrm{av}}\right)+1\right)\right.} \prod_{j=1}^{n}\left[\frac{2}{1+\gamma_{j}} \Gamma\left(1-H\left(1+\gamma_{j}\right)\right)\right]
\end{aligned}
$$

where $f: \mathbb{R}_{+}^{n} \times \mathbb{R}^{n} \rightarrow(0, \infty)$ is given by

$$
f(t, y)=t_{1}^{H}\left|y_{1}\right| \vee t_{2}^{H}\left|y_{2}\right| \vee \cdots \vee t_{n}^{H}\left|y_{n}\right|,
$$

and (2.6) is established.

Acknowledgments. G. Kerchev and I. Nourdin are supported by the FNR OPEN grantAPOGee at Luxembourg University.

\section{References}

[1] F. Aurzada and C. Mönch (2018): Persistence probabilities and a decorrelation inequality for the Rosenblatt process and Hermite processes. Teor. Veroyatn. Primen 63, no. 4, pp. 817-826

[2] A. Ayache, D. Wu and Y. Xiao (2008): Joint continuity of the local times of fractional Brownian sheets. Ann. Inst. H. Poincaré Probab. Statist. 44, no. 4, pp. 727-748.

[3] S.M. Berman (1969): Local times and sample function properties of stationary Gaussian processes. Trans. Amer. Math. Soc. 137, pp. 277-299.

[4] R. Bhatia (1997): Matrix analysis. Volume 169 of Graduate Texts in Mathematics, SpringerVerlag, New York.

[5] A. Chronopoulou, F.G. Viens and C.A. Tudor (2009): Variations and Hurst index estimation for a Rosenblatt process using longer filters. Electron. J. Stat. 3, pp. 1393-1435.

[6] R.L. Dobrushin and P. Major (1979): Non-central limit theorems for nonlinear functionals of Gaussian fields. Z. Wahrsch. Verw. Gebiete 50, pp. 27-52.

[7] M.R. Dostanić (1998): Spectral properties of the operator of Riesz potential type. Proc. Amer. Math. Soc. 126, pp. 2291-2297.

[8] M. Dozzi (2003): Occupation density and sample path properties of $N$-parameter processes. In Topics in spatial stochastic processes (Martina Franca, 2001), volume 1802 of Lecture Notes in Math., pp. 127-166. Springer, Berlin, 2003.

[9] J. Duoandikoetxea (2001): Fourier analysis, volume 29 of Graduate Studies in Mathematics, American Mathematical Society, Providence, RI. Translated and revised from the 1995 Spanish original by David Cruz-Uribe.

[10] A. M. Garsia, E. Rodemich and H. Rumsey Jr. (1970): A real variable lemma and the continuity of paths of some Gaussian processes. Indiana Univ. Math. J. 20, pp. 565-578.

[11] L. Grafakos (2008): Classical Fourier Analysis, Graduate Texts in Mathematics, Springer, New York. 
[12] L. Grafakos (2009): Modern Fourier Analysis, Graduate Texts in Mathematics, Springer, New York.

[13] Y. Gu and G. Bal (2012): Random homogenization and convergence to integrals with respect to the Rosenblatt process. J. Differential Equations 253, no. 4, pp. 1069-1087.

[14] R. Hunt, B. Muckenhoupt and R. Wheeden (1973): Weighted norm inequalities for the conjugate function and Hilbert transform. Trans. Amer. Math. Soc. 176, pp. 227-251.

[15] O. Kallenberg (2002): Foundations of modern probability. Probability and its Applications (New York), 2nd edition. Springer-Verlag, New York.

[16] I. Nourdin and D. Tran (2019): Statistical inference for Vasicek-type model driven by Hermite processes. Stochastic Process. Appl. 129, no. 10, pp. 3774-3791.

[17] M. Reed and B. Simon (1980): Methods of modern mathematical physics. I. Academic Press, Inc. [Harcourt Brace Jovanovich, Publishers], New York. Second edition.

[18] C.A. Rogers and S.J. Taylor (1961). Functions continuous and singular with respect to a Hausdorff measure, Mathematika 8, pp. 1-31.

[19] M. Rosenblatt (1961): Independence and dependence. In Proc. 4th Berkeley Sympos. Math. Statist. and Prob., Vol. II, pp. 431-443. Univ. California Press, Berkeley, Calif.

[20] G. Shevchenko (2010): Properties of trajectories of the multifractional Rosenblatt process. Teor. Imovīr. Mat. Stat. 83, pp. 138-147.

[21] Y. Xiao (1997): Hölder conditions for the local times and the Hausdorff measure of the level sets of Gaussian random fields. Probab. Theory Rel. Fields 109, pp. 129-157.

University of Luxembourg, Department of Mathematics, Luxembourg

E-mail address: george.kerchev@uni.lu

University of Luxembourg, Department of Mathematics, Luxembourg

E-mail address: ivan.nourdin@uni.lu

University of Helsinki, Department of Mathematics and Statistics, Finland

E-mail address: eero.saksman@helsinki.fi

Aalto University School of Business, Department of Information and Service MANAGEMEnt, Finland

E-mail address: lauri.viitasaari@iki.fi 